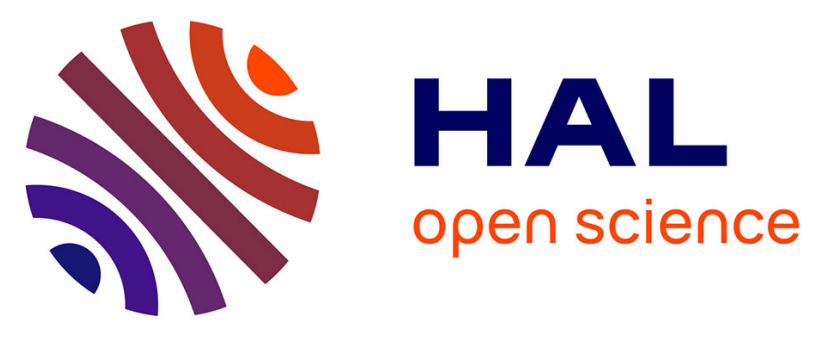

\title{
Design of New Wavelet Packets Adapted to High-Resolution SAR Images With an Application to Target Detection
}

Ammar Mian, Jean-Philippe Ovarlez, Abdourrahmane Atto, Guillaume Ginolhac

\section{To cite this version:}

Ammar Mian, Jean-Philippe Ovarlez, Abdourrahmane Atto, Guillaume Ginolhac. Design of New Wavelet Packets Adapted to High-Resolution SAR Images With an Application to Target Detection. IEEE Transactions on Geoscience and Remote Sensing, 2019, 57 (6), pp.3919-3932. $10.1109 /$ tgrs.2018.2888993 . hal-02057622

\section{HAL Id: hal-02057622 \\ https://hal.univ-grenoble-alpes.fr/hal-02057622}

Submitted on 1 Jul 2019

HAL is a multi-disciplinary open access archive for the deposit and dissemination of scientific research documents, whether they are published or not. The documents may come from teaching and research institutions in France or abroad, or from public or private research centers.
L'archive ouverte pluridisciplinaire HAL, est destinée au dépôt et à la diffusion de documents scientifiques de niveau recherche, publiés ou non, émanant des établissements d'enseignement et de recherche français ou étrangers, des laboratoires publics ou privés. 


\title{
Design of New Wavelet Packets Adapted to High-Resolution SAR Images with an Application to Target Detection
}

\author{
Ammar Mian ${ }^{1,2}$, Student Member, IEEE, Jean-Philippe Ovarlez ${ }^{1,3}$, Member, IEEE, Abdourrahmane M. ATto ${ }^{2}$, \\ Senior Member, IEEE, Guillaume GINOLHAC ${ }^{2}$, Member, IEEE
}

\begin{abstract}
High-Resolution in synthetic aperture radar (SAR) leads to new physical characterizations of scatterers which are anisotropic and dispersive. These behaviors present an interesting source of diversity for target detection schemes. Unfortunately, such characteristics have been integrated and have been naturally lost in monovariate single look SAR images. Modeling this behavior as non-stationarity, wavelet analysis has been successful in retrieving this information. However, the sharp-edge of the used wavelet functions introduces undesired high side-lobes for the strong scatterers present in the images. In this paper, a new family of parametrized wavelets, designed specifically to reduce those side-lobes in the SAR image decomposition, is proposed. Target detection schemes are then explored using this spectroangular diversity and it can be shown that in High-Resolution SAR images, the non-Gaussian and robust framework leads to better results.
\end{abstract}

Index Terms-Synthetic Aperture Radar ; Wavelet Packets ; Robust Adaptive Detection ; High-resolution

\section{INTRODUCTION}

\section{A. Motivations}

$\mathbf{R}$ ADAR systems play a major role in modern military applications, air and ground traffic control, autonomous vehicles, observation of earth and other planetary systems, monitoring of dynamic objects such as forests, glaciers, meteors, etc. An intelligent operational radar vision system involves self-tuned parameterization of the radar waveform emission (pulse-width and repetition interval, transmitter power, etc.) and optimal target detection/characterization for automatic perception of a monitored environment. This requires selfcoordination of the transmitter and receiver which can operate in open or close loops. This also requires integrating in radar based machine intelligence frameworks, the capability of learning new/unknown radar waveforms. On the contrary, when considering earth observation from satellites using Synthetic Aperture Radars (SAR), mainly artificial intelligence frameworks are developed in order to process the data obtained for various applications.

SAR are moving radars systems capable of producing highquality images of the earth's surface [1]. They consist in emitting an electromagnetic wave that is reflected on the

${ }^{1}$ SONDRA, CentraleSupélec, Université Paris-Saclay, F-91190, Gif-surYvette, France.

${ }^{2}$ Université Savoie Mont Blanc, LISTIC, France

3 DEMR, ONERA, Université Paris-Saclay, F-91123, Palaiseau, France.

The work was partially supported by PHOENIX ANR-15-CE23-0012 grant of the French National Agency of Research. earth's surface. The backscattered signal, which is affected by the scatterers on the surface, is then processed to build an image of the scene. SAR systems are known for their usability in all weather and illumination situations. They are thus capable to monitor all kind of areas of interest. Recent years have seen an increase in the number of SAR systems.Missions such as Sentinel-1 or TerraSAR-X have for example a global coverage of the earth's surface with SAR electronic imaging technology.

New missions in peculiar have a large bandwidth and an increased spatial resolution. In this context, specific responses of the scatterers have been observed. They are anisotropic and dispersive [2], [3] in contrast to the usual isotropic and whiteness assumption made in traditional SAR reconstruction algorithms. When considering HR monovariate SAR images, a dispersion of the energy is observed. A single scatterer may have many side lobes on the reconstructed image. Moreover, the specific spectro-angular response of the scatterer is naturally lost during the conventional SAR processing. This information is of interest and can be exploited as an additional source of diversity in many applications.

In SAR images processing, depending on the application, three important techniques are usually relied upon: target detection, segmentation and classification. Target Detection schemes [4] consist in deciding if a target of interest is present at a given position of the image. Segmentation [5], [6], aim's is to delimit the image into segments which are conceptually meaningful such as the boundary between land and sea. Finally classification [7], [8], [9], [10] allows to label part of the images with regards to an application of interest. This paper focuses on target detection schemes and more specifically, on schemes which respect the Constant False Alarm (CFAR) property [11]. This property allows to detect targets while guaranteeing a certain probability of false alarm.

\section{B. Relation to prior works}

Classic schemes usually rely on a diversity of some sort to characterize the target and to separate it from the clutter. Literature on the subject concerning SAR images is vast as many kind of diversities can be exploited. For example, in [4], a spatial template model of target is used in likelihood ratio schemes to derive detectors. Polarimetric diversity has been used in [12], [13] to detect vehicles under foliage, in [14] to detect ship in sea clutter or in [15], [16] for segmentations 
purposes. In, [17] a diversity coming from successive pulses is used to detect range-spread targets. In [18], [19], [20], a timedivision multiplexing of the antennas is exploited to create a diversity for Ground Moving Targets Identification (GMTI). An extension to non Gaussian model has been explored in [21], [22], [23].

In this paper, the spectro-angular behaviour mentionned earlier as a source of diversity is considered. Several work have investigated methods for retrieving the spectro-angular diversity. For example, approach such as steerable pyramids [24], [25], curvelets [26] or subspaces [27], [28] are possible. However, they are usually heavy methods. For example, subspace methods have high-computational cost and are not adaptive. They also assume the knowledge of a physical model, which makes them specific to an application. Time frequency analysis is a simpler approach that allows to analyze SAR data to retrieve non-stationary information such as spectral and angular behaviors. This approach was used in [29], where the azimuth bandwidth has been separated in two sub-bands for ship detection. In the following we restrict only on the Linear Time Frequency Distributions (LTFD) as they allow to keep the phase information and the possibility to apply the reconstruction property.

Wavelet decomposition of SAR images have been studied for many applications. In [30], [31], wavelet frames have been used in order to derive a measure of polarimetric texture used in segmentation and target detection schemes. Wavelet transforms on SAR images have been used in [32] to retrieve wind fields. In [33], wavelets have been used to reduce speckle noise in interferometric SAR images. Fusion techniques on wavelet coefficients have been used in [34] in order to compute a change detection map. Wavelet decomposition associated with kurtosis statistics have been exploited in [6], [7] for both segmentation and classification purposes. In [35], [36], multiresolution information is used for target detection schemes in Gaussian context. Retrieval of spectro-angular diversity using wavelet decomposition have in peculiar been investigated in work such as [37], [38]. More precisely, these methods have been used for target detection applications in [39] or for change detection in [40]. In those works, the spectro-angular information has presented promising results. However, in those works the decomposition induced side lobes on the sub images which may decrease the performance.

This paper proposes a wavelet packet formalism (as a generalization of LTFD) and designs a new family of wavelets aimed at decreasing these side lobes. Then, the multivariate image resulting from the wavelet decomposition is modeled by a multivariate statistical framework. This framework allows to retrieve the properties of the clutter through a covariance matrix parameter. Using this modeling, classic adaptive methods can be used in order to detect a target embedded in clutter disturbances. The spectro-angular behaviour of the target is assumed to be known through a steering vector. These adaptive methods have been extensively studied in [41], [42], where the disturbances are modeled as a realization of a Gaussian random variable parametrized by an unknown covariance matrix. Classically, secondary data, assumed to be free of target, corresponding to surrounding pixels are used for the estimation of the covariance matrix. Since in this paper the images are the result of a wavelet decomposition, the size of the vector is expected to be large. Clearly, when the size of the vectors is large, the number of secondary data needed increases. This is problematic in HR SAR images where a heterogeneity of the data is naturally present. In this case, the hypothesis of homogeneity of the surrounding pixels is not evident and the Gaussian model reflect poorly the observations. Robust methods have been developed using broader families of distributions than the Gaussian one [43], [44]. In this paper, both Gaussian and robust methods will be studied and compared.

\section{Contributions of the present paper}

The different contributions of the present paper are summarized as follows:

- A parametrized wavelet family is proposed to analyze SAR Images. The new family is an extension of Shannon M-Band filters that are adapted to take into account SAR geometry. The new family is designed to reduce side lobes on the wavelet coefficients. A criteria is proposed for the choice of its parameters.

- The spectro-angular diversity, obtained through wavelet decomposition, is used in robust target detection schemes. Target detection schemes are explored in both Gaussian and elliptical noise assumption and are studied through two alternatives CFAR detectors: the Adaptive Matched Filter (AMF) and the Adaptive Normalized Matched Filter (ANMF). The behaviour of those detectors in terms of regulation of false alarm and performance of detection are compared using two separate datasets.

\section{Paper Organization}

The paper is organized as follows: in section II, the acquisition geometry of SAR is recalled and it is explained how the information about anisotropy and dispersivity of scatterers is lost in the processing of the single look monovariate image. Then, in section III, Shannon M-band wavelets are adapted for the purpose of retrieving spectro-angular diversity in SAR images. In section IV, an application of wavelet decomposition to target detection is presented and in section $\mathrm{V}$, simulations and results are presented. Finally, some conclusions are drawn in section VI. Proofs are given in Appendices.

The following conventions are adopted: matrices are in bold and capital, vectors in bold. $\mathbb{R}^{N}$ and $\mathbb{C}^{N}$ refer to the sets of N-dimensional real and complex vectors. $L^{n}\left(\mathbb{C}^{2}\right)$ is the set of the functions having values in $\mathbb{C}^{2}$ for which the $n$ th power of the absolute value is integrable. For a given complex scalar, - denotes the conjugate operator, $|\bullet|$ is the module operator. For any given vector or matrix, $\bullet^{T}$ denotes the transpose operator, $\bullet H$ denotes the transpose conjugate operator, $\|\bullet\|$ is the Euclidean norm. For any matrix | $\mid$ denotes the determinant. Given a 2-dimensional (2D) function $g \in L^{1}\left(\mathbb{C}^{2}\right) \cup L^{2}\left(\mathbb{C}^{2}\right)$, the 2D Fourier transform (resp. inverse Fourier Transform) is denoted by $\mathcal{F} g\left(\omega_{1}, \omega_{2}\right)=$ $\int_{\mathbb{R}} g\left(x_{1}, x_{2}\right) e^{-i \omega_{1} x_{1}} e^{-i \omega_{2} x_{2}} \mathrm{~d} x_{1} \mathrm{~d} x_{2}$ (resp. $\mathcal{F}^{-1} g$ ) and define $\tau_{[p, q]} g(x, y)=g(x-p, y-q)$. For a function, $\langle\bullet, \bullet\rangle$ is the 
inner product on $L^{1}\left(\mathbb{C}^{2}\right) \cup L^{2}\left(\mathbb{C}^{2}\right)$ and $\|\bullet\|$ is the $L^{2}$ norm. $\mathbb{1}_{K}$ denotes the indicator function of a given set $K$.

\section{SAR IMAgE AND Non-Stationarities}

In this section, we describe the geometry of acquisition for a SAR system and give the definition of relevant physical parameters. We then explain how algorithms such as Range Migration Algorithm (RMA) result in a loss of information when considering HR SAR image.

\section{A. SAR Acquisition Geometry}

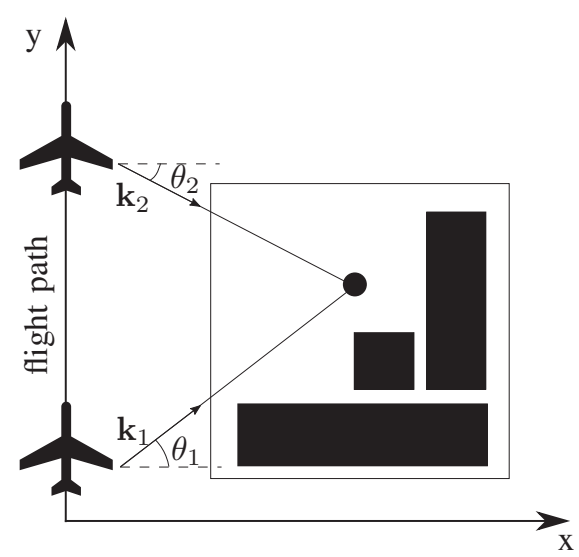

Fig. 1. SAR acquisition geometry. A reflector is viewed at two different angles of illumination $\theta_{1}$ and $\theta_{2}$ in a given frequency. This information is summarized through the wave vectors $\mathbf{k}_{1}, \mathbf{k}_{2}$.

Figure 1 presents the geometry of acquisition for a SAR system. The moving radar transmits an electromagnetic wave represented by the wave vector $\mathbf{k}=\left[k_{x}, k_{y}\right]^{T}$ and recovers the backscattering signal in order to obtain a map of the reflectors of the scene. $\mathbf{k}$ is related to the emitted frequency $f$ by $\|\mathbf{k}\|=$ $\mathfrak{K}=2 f / c, c$ being the celerity of the light, and to the angle of illumination $\theta$ by $\theta=\arctan \left(k_{y} / k_{x}\right)$.

The emitted signal is located in a certain range of frequencies defined by: $\left[f_{0}-B / 2, f_{0}+B / 2\right], f_{0}$ being the carrier and $B$ being the bandwidth of the radar. This translates in terms of spatial frequencies $\mathfrak{K}$ to: $\left[\mathfrak{K}_{0}-\mathfrak{K}_{B} / 2, \mathfrak{K}_{0}+\mathfrak{K}_{B} / 2\right]$ with $\mathfrak{K}_{0}=2 f_{0} / c, \mathfrak{K}_{B}=2 B / c$. The angles $\theta$ of illumination lies in $\left[-\theta_{B}, \theta_{B}\right]$ angular domain. The spatial SAR resolutions are given respectively in azimuth by $\delta_{y}=c /\left(4 f_{0} \theta_{B}\right)$ and in radial range by $\delta_{x}=c /(2 B)$. We define $\mathcal{D}=$ $\left[\mathfrak{K}_{0}-\mathfrak{K}_{B} / 2, \mathfrak{K}_{0}+\mathfrak{K}_{B} / 2\right] \times\left[-\theta_{B}, \theta_{B}\right]$, and $\mathbf{U}^{\mathbf{S}, \measuredangle}$ as the space of functions having spectro-angular features in $\mathcal{D}$.

\section{B. Anisotropy and Dispersivity of Scatterers}

In HR SAR Images, the hypothesis of isotropy and nondispersivity of the scatterers is no longer obvious. When a target is illuminated using a large bandwidth and a large range of angles, it is more reasonable to assume that its response is dependent of the wave vector. Recent studies of the spectral and angular behaviour of the scatterers have shown the variation of the scatterers' response for several angles of illumination and several frequencies [2], [45].

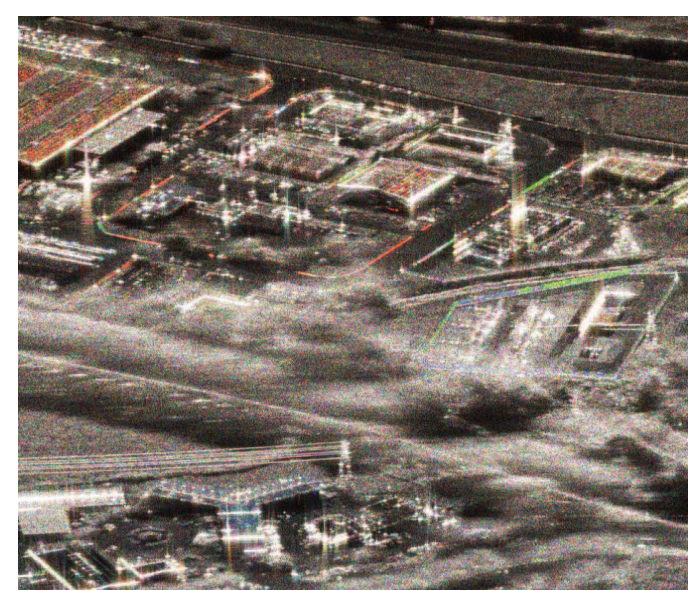

Fig. 2. An ONERA RGB color-coded SAR image acquired for three consecutive frequency bands.

Figure 2 presents an ONERA SAR image in X-band. The responses of the scene relative to three consecutive frequency bands have been coded in RGB color-coding. Red points are responding only on the first band, green ones on the second band and the blue ones on the third band. They are called colored scatterers. Gray points are called white scatterers as they are responding equivalently in the three sub-bands. This image perfectly illustrates how some scatterers have different behaviour given the band used. Similar results can be achieved when looking the scene at different range of angles. This diversity is of interest as it can be interpreted in terms of target characteristic: given the spectro-angular behaviour of an object of interest, one can adapt target detection schemes on multivariate SAR images to this specific behaviour.

Unfortunately, for complex monovariate SAR images, this information is lost during the processing. In algorithms such as RMA [1], the aim is to collect a backscattering reflection coefficient $\tilde{I}(\mathbf{k})$ and then perform Fourier based spectral estimation in order to build the conventional complex single look (monovariate) SAR image $I(\mathbf{r})$ for each point $\mathbf{r}=[x, y]^{T}$ on the ground:

$$
I(\mathbf{r})=\int_{\mathcal{D}} \tilde{I}(\mathbf{k}) \exp \left(2 i \pi \mathbf{k}^{T} \mathbf{r}\right) d \mathbf{k}
$$

where the integration is performed on the whole spectral and angular domains. When colored scatterers are present, their spectro-angular behaviour is effectively lost.

Works such as [37] or [38] have proposed to model the nonstationarities of the scatterers of the image $I$ in the space of spectral and angular features $[\mathfrak{K}, \theta]$. Using this model, wavelet analysis is a powerful tool for analyzing the behaviour of the colored scatterers. For instance, an hyperimage representing the reflectivity of the scene for any sub-space $\mathcal{E} \subset \mathcal{D}$ is given by:

$$
\tilde{I}_{\mathcal{E}}(\mathbf{r})=\int_{\mathcal{E}} \tilde{I}(\mathbf{k}) \overline{\Psi_{\mathcal{E}}^{\mathbf{S}}}(\mathbf{k}, \mathbf{r}) d \mathbf{k}
$$

where $\Psi_{\mathcal{E}}^{\mathbf{S}}(\mathbf{k}, \mathbf{r})$ is a wavelet function with spectro-angular support $\mathcal{E}$. When considering several subsets $\mathcal{E}_{1, \ldots, M}$, a wavelet 
packet $\left\{\Psi_{\mathcal{E}_{i}}^{\mathbf{S}}(\mathbf{k}, \mathbf{r}) / i=1, \ldots, M\right\}$ can be defined. The problematic is then to choose the shape of the wavelets and a relevant partition of $\mathcal{D}$ in terms of $\mathcal{E}_{i}$ as to decompose the image in separate frequency bands and range of angles for a given purpose.

This approach was used in [39], [40] where good results in both target detection and change detection have been obtained. However, in those works, the problem of side lobes has not been considered. We propose in the next section to design new wavelets aimed at reducing the dispersion of energy on the sub images.

\section{NeW WAVELET DESIGN FOR SAR ANALYSis}

In this section, new wavelet packets adapted to SAR geometry are developed. The particularity of this new packet with regards to existing literature is the choice of polar representation which better describes the data with regards to the spectro-angular diversity of interest. To this end, we use classic Shannon M-band filters that we adapt to take into account the spectral support of SAR Images and then we correct the edge effects of those wavelets.

The adaptation of wavelet packets in this geometry can be done using many classic wavelets packets (Gabor, Debaucheries, etc.). However, Shannon M-band filters have been chosen as a basis of our design for the following reasons:

- They are separable with regards to the two dimensions $(\mathfrak{K}, \theta)$ of the decomposition, which makes them ideal when we want to choose the number of sub-bands and sub-looks (as in looking angle) separately.

- Since we expect to exploit the decomposition in classic target detection scheme, there is a need for each coefficient to deliver a different information than the others. Otherwise, correlations between sub-bands/sublooks would be introduced due to the shape of the wavelets and may deteriorate detection performance. This leads to a choice of an orthogonal wavelet packet.

- To better describe the behaviour of a possible target as a function of the frequencies and looking angle, we consider wavelets corresponding to a connected subset of the frequency/angular domain.

\section{A. Shannon M-band wavelets theory}

Let $M_{1}$ and $M_{2}$ be natural numbers that are both greater than or equal to 2. The Shannon 2D $M_{1} \times M_{2}$ multi-band wavelet filters used in this paper follow from a separable 2D extension of 1D filters presented in [46], [47]. These filters give a multi-resolution framework for decomposing any image.

We define $\mathbf{U}^{S}$ as the 2D Paley-Wiener (PW) space composed by elements of $L^{2}\left(\mathbb{R}^{2}\right)$ whose Fourier transform is supported within $[-\pi, \pi]^{2}$. Any element of this space satisfies Shannon's sampling theorem. Therefore, when the $M_{1} \times M_{2}$ multi-band decomposition concerns the $\mathrm{PW}$ space $\mathbf{U}^{\mathrm{S}}$, the input data for the decomposition of any element $g$ of this functional space are the samples $\{g[k, \ell]\}_{k, \ell \in \mathbb{Z}}$ of $g$ (corresponding to the pixels of the image to decompose).
The 2D Shannon wavelet packet function at resolution level $j$ and 2D shift parameters $\left(n_{1}, n_{2}\right)$, with $n_{\varepsilon} \in\left\{0, \ldots, M_{\varepsilon}^{j}-1\right\}$ for $\varepsilon \in\{1,2\}$, is given by

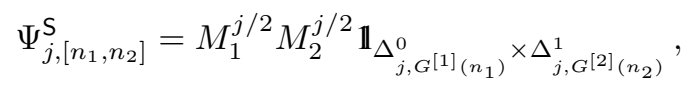

where

$$
\Delta_{j, k}^{\varepsilon}=\left[-\frac{(k+1) \pi}{M_{\varepsilon}^{j}},-\frac{k \pi}{M_{\varepsilon}^{j}}\right] \cup\left[\frac{k \pi}{M_{\varepsilon}^{j}}, \frac{(k+1) \pi}{M_{\varepsilon}^{j}}\right],
$$

and $\left(G^{[\varepsilon]}\right)_{\varepsilon \in\{1,2\}}$ are the permutation maps defined respectively, for $\varepsilon \in\{1,2\}$, by $G^{[\varepsilon]}(0)=0$ and by recursively setting, for $k=0,1, \ldots, M_{\varepsilon}-1$ and $\ell=0,1,2, \ldots$

$$
\begin{aligned}
G^{[\varepsilon]} & (M \ell+k) \\
& = \begin{cases}M G^{[\varepsilon]}(\ell)+k & \text { if } G^{[\varepsilon]}(\ell) \text { is even, } \\
M G^{[\varepsilon]}(\ell)-k+M-1 & \text { if } G^{[\varepsilon]}(\ell) \text { is odd. }\end{cases}
\end{aligned}
$$

Define $\Phi_{j,\left[n_{1}, n_{2}\right]}^{\mathrm{S}}=\mathcal{F}^{-1} \Psi_{j,[m, n]}^{\mathrm{S}}$. A 2D wavelet packet subspace $\boldsymbol{\Phi}_{j,\left[n_{1}, n_{2}\right]}^{\mathrm{S}}$ is generated as the closure of the space spanned by the following translated versions of $\Phi_{j,\left[n_{1}, n_{2}\right]}^{\mathrm{S}}$ :

$$
\mathbf{\Phi}_{j,[m, n]}^{\mathbf{S}}=\operatorname{Clos}\left\langle\tau_{\left[M_{1}^{j} p, M_{2}^{j} q\right]} \Phi_{j,[m, n]}^{\mathbf{S}}: p \in \mathbb{Z}, q \in \mathbb{Z}\right\rangle .
$$

These subspaces are such that for any fixed $j$,

$$
\mathbf{U}^{\mathrm{S}}=\bigoplus_{\substack{m=0,1, \ldots, M_{1}^{j}-1 \\ n=0,1, \ldots, M_{2}^{j}-1}} \boldsymbol{\Phi}_{j,[m, n]}^{\mathrm{S}}
$$

where $\oplus$ denotes the direct sum of functional subspaces.

As an illustration, the Shannon $2 \times 3$ multi-band wavelet packet tree is given by Figure 3 as a tree product resulting from a 2-band and a 3-band 1D trees, where the tree-product involves all combination of nodes given at a fixed resolution level $j$. In this figure, the positive part $\Delta_{j, k}^{\varepsilon,+}$ of $\Delta_{j, k}^{\varepsilon}$ is given for each resolution level $j$ under consideration.

The Shannon $M_{1} \times M_{2}$ multi-band coefficients of the projection of $g$ on a 2D wavelet packet subspace $\boldsymbol{\Phi}_{j,\left[n_{1}, n_{2}\right]}^{\mathrm{S}}$ defines the wavelet coefficients:

$$
\mathbf{C}_{j,\left[n_{1}, n_{2}\right]}^{\mathrm{S}}[p, q]=\iint_{\mathbb{R}^{2}} g(z, t) \tau_{\left[M_{1}^{j} p, M_{2}^{j} q\right]} \Phi_{j,\left[n_{1}, n_{2}\right]}^{\mathrm{S}}(z, t) \mathrm{d} z \mathrm{~d} t .
$$

Proposition III.1. The coefficients of the projection of $g$ on a wavelet packet subspace $\boldsymbol{\Phi}_{j,\left[n_{1}, n_{2}\right]}^{\mathrm{S}}$ is a discrete sequence $\mathbf{C}_{j,\left[n_{1}, n_{2}\right]}=\left(\mathbf{C}_{j,\left[n_{1}, n_{2}\right]}^{\mathrm{S}}[p, q]\right)_{p, q \in \mathbb{Z}}$ where

$$
\mathbf{C}_{j,[m, n]}^{\mathrm{S}}[p, q]=M_{1}^{-j / 2} M_{2}^{-j / 2} \mathcal{F}^{-1} U_{j,[m, n]}\left(M^{j} p, M^{j} q\right),
$$

with

$$
U_{j,[m, n]}=[\mathcal{F} g] \times \mathbb{1}_{\Delta_{j, G(m)}^{0} \times \Delta_{j, G(n)}^{1}} .
$$

Proof. See Appendix A.

In practice, $g$ is a discrete image to be decomposed. This proposition shows how wavelet coefficients can be easily obtained in practice through a simple Fast Fourier Transform (FFT). $[p, q]$ are the pixels of the wavelet coefficient for shift parameters $\left[n_{1}, n_{2}\right]$ at a fixed resolution $j$. Note that, a decomposition at a given resolution $j$ assume that the wavelets 

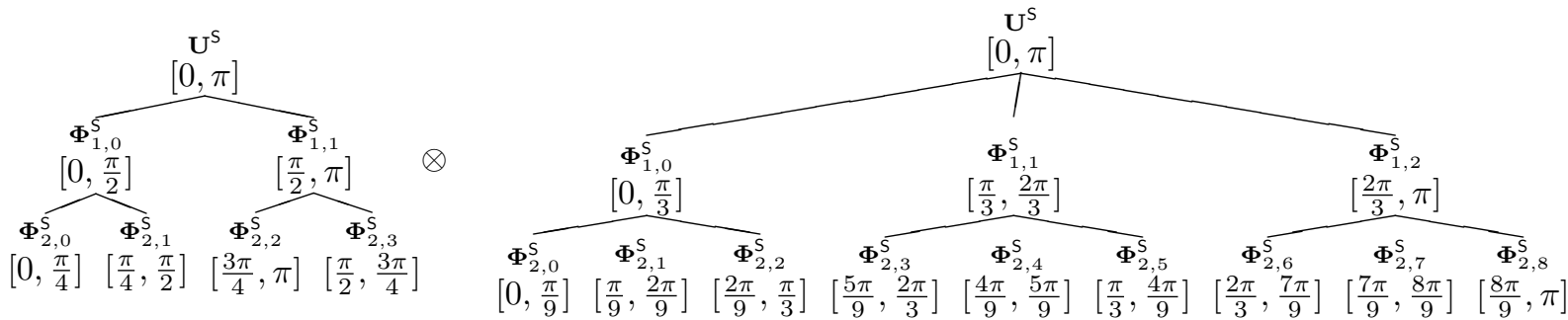

Fig. 3. Shannon $2 \times 3$ multi-band decomposition tree associated with $j=2$. The positive part of $\Delta_{j, \bullet}^{1,+}$ of $\Delta_{j, \bullet}^{1}$ are given in the left-side tree whereas $\Delta_{j, \bullet}^{2,+}$, positive part of $\Delta_{j, \bullet}^{2}$ are given at the right-side tree. The frequency tiles associated with the decomposition are the intervals $\Delta_{j, \bullet}^{1} \times \Delta_{j, \bullet}^{2}$ for every fixed $j$ : the whole tree involves all combination of nodes given at a fixed resolution level $j$.
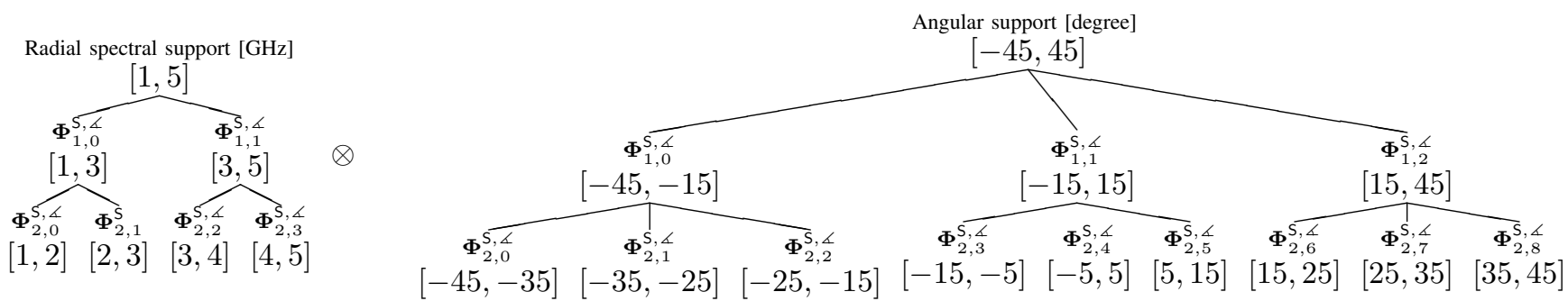

Fig. 4. Cartesian product $\otimes$ of spectral and angular multi-resolution trees associated with radial ' 2 bands - 2 resolutions' and angular ' 3 looks - 2 resolutions' splitting $\Psi_{j,[m, n]}^{\mathrm{S}, L}(\mathfrak{K}, \theta)$ defined by Eq. (16) when considering $\left[f_{0}-B / 2, f_{0}+B / 2\right] \times\left[-\theta_{B}, \theta_{B}\right]=[1 G H z, 5 G H z] \times[-45 \mathrm{deg}, 45 \mathrm{deg}]$. The intervals represented are given for illustration.

coefficients correspond to a decimated version of the image (by a factor of $M_{1}^{j}$ and $M_{2}^{j}$ ). This methodology allows to compute efficiently the coefficients with a low-complexity. Indeed, since only a Hadamard product and a FFT is necessary, the complexity is linear with regards to the number of coefficients desired.

The wavelets presented here are designed for images respecting Shannon sampling theorem. The decomposition is done on functions whose frequencies are contained in the space $[-\pi, \pi]^{2}$. We adapt hereafter Shannon wavelets from the Cartesian space $[-\pi, \pi]^{2}$ to the polar space $\mathcal{D}$ corresponding to the physical diversity of interest for SAR images.

\section{B. Adaptation of Shannon wavelets to SAR Geometry}

Define

$$
\Psi_{j,[m, n]}^{\mathbf{S}, \measuredangle}(\mathfrak{K}, \theta)=R^{j / 2} L^{j / 2} \mathbb{1}_{\Delta_{j, \kappa_{m}} \times \Delta_{j, \theta_{n}}}(\mathfrak{K}, \theta),
$$

where

$$
\begin{aligned}
\Delta_{j, \mathfrak{K}_{m}} & =\mathfrak{K}_{0}-\mathfrak{K}_{B}+\left[\frac{m \mathfrak{K}_{B}}{R^{j}}, \frac{(m+1) \mathfrak{K}_{B}}{R^{j}}\right], \\
\Delta_{j, \theta_{n}} & =\left[\frac{n \theta_{B}}{L^{j}}, \frac{(n+1) \theta_{B}}{L^{j}}\right] .
\end{aligned}
$$

From this, we define the wavelet functions $\Phi_{j,[m, n]}^{\mathbf{S}, \angle}(x, y)=$ $\mathcal{F}^{-1} \Psi_{j,[m, n]}^{\mathbf{S}, L}(\mathfrak{K}, \theta)$. Here, the variables $x$ and $y$ correspond to the range and cross-range position as in figure 1. Note that this definition requires computing the Fourier transform on spectral and angular variables. Among the different possible solutions of this problem, we will use interpolation from the Fractional Fast Fourier Transform (FFFT or 3FT) in order to fill the Polar grid $\left[\mathfrak{K}_{0}-\mathfrak{K}_{B} / 2, \mathfrak{K}_{0}+\mathfrak{K}_{B} / 2\right] \times\left[-\theta_{B}, \theta_{B}\right]$ from the Cartesian one corresponding to variables $k_{x}, k_{y}$. Among the 3FT implementations, we recommend using that of [48].
The wavelets thus defined constitute a wavelet packet as per the following proposition;:

Proposition III.2 (Vanishing moments). For any non-negative integers $j, m, n, p, q$, we have

$$
\iint_{\mathbb{R}^{2}} x^{p} y^{q} \Phi_{j,[m, n]}^{\mathbf{S}, \measuredangle}(x, y) d x d y=0 .
$$

Proof. See Appendix B.

Functions $\Phi_{j,[m, n]}^{\mathbf{S}, \angle}$ defined above have thus an infinite number of vanishing moments. Since they are well localized in space/frequency/angle, they are wavelet functions.

Define the wavelet subspaces $\Phi_{j,[m, n]}^{\mathbf{S}, L}$ similarly to eq. (6). Then we have:

Proposition III.3 (Orthogonality of wavelet packet subspaces). For any given $j$ and any $(m, n) \neq\left(m^{\prime}, n^{\prime}\right)$ we have:

$$
\mathbf{\Phi}_{j,[m, n]}^{\mathbf{S}, \angle} \perp \mathbf{\Phi}_{j,\left[m^{\prime}, n^{\prime}\right]}^{\mathbf{S}, \angle},
$$

where $\perp$ denotes orthogonality symbol.

Proof. See Appendix B.

Proposition III.4 (Completion of wavelet packet subspaces). For any given $j$, and any $(m, n)$, we have

$$
\bigcup_{\substack{m=0,1, \ldots, R^{j}-1 \\ n=0,1, \ldots, L^{j}-1}} \boldsymbol{\Phi}_{j,[m, n]}^{\mathbf{S}, L}=\mathbf{U}^{\mathbf{S},, L} .
$$

Proof. See Appendix B.

Propositions III.2, III.3 and III.4 highlight that wavelet subspaces $\boldsymbol{\Phi}_{j,[m, n]}^{\mathrm{S}, L} \quad: \quad j \geqslant$ $1, m=0,1, \ldots, R^{j}-1, n=0,1, \ldots, L^{j}-1 \quad$ can $\quad$ thus 
be used to define several multi-resolution frameworks (specific sub-selection of $j, m, n$ ) for analyzing SAR data.

Analyzing SAR data is done by computing the wavelet coefficients as previously stated in (2). We have:

Proposition III.5 (Wavelet coefficients). In practice, at a resolution level $j$, we obtain the wavelet coefficients by computing the following:

$$
\mathbf{C}_{j,[m, n]}^{\mathrm{S}, L}[p, q]=R^{-j / 2} L^{-j / 2} \mathcal{F}^{-1} V_{j,[m, n]}\left(R^{j} p, L^{j} q\right),
$$

where $V_{j,[m, n]}$ follows from the back-projection of the SAR image with respect to spectral and illumination features:

$$
V_{j,[m, n]}(\mathfrak{K}, \theta)=\widetilde{I}(\mathfrak{K}, \theta) \mathbb{1}_{\Delta_{j, \mathfrak{K}_{m}} \times \Delta_{j, \theta_{n}}}(\mathfrak{K}, \theta) .
$$

\section{Proof. Similar to proposition III.1.}

Again, the complexity of this methodology is linear with regards to the number of coefficients $(R \times L)$ since coefficients are obtained from a Hadamard product and a 3FT. In practice, any SAR data can efficiently analyzed using this methodology.

An example of a multi-resolution analysis is given in Figure 4 for spectral features and angular illumination in $\left[f_{0}-B / 2, f_{0}+B / 2\right] \times\left[-\theta_{B}, \theta_{B}\right]=[1,5] \mathrm{GHz} \times$ $[-45,45]$ deg.

\section{Bell-Shaped wavelets design for SAR Geometry}

When considering Shannon wavelets, the decomposition is subject to hard transition in the sense that each filter is an ideal band-pass filter. When considering the wavelet coefficients, this results in a convolution with a sinc function which has high side lobes (see fig. 5 for an illustration). This dispersion of energy is problematic in detection schemes when secondary data, corresponding to the surrounding pixels, are needed.
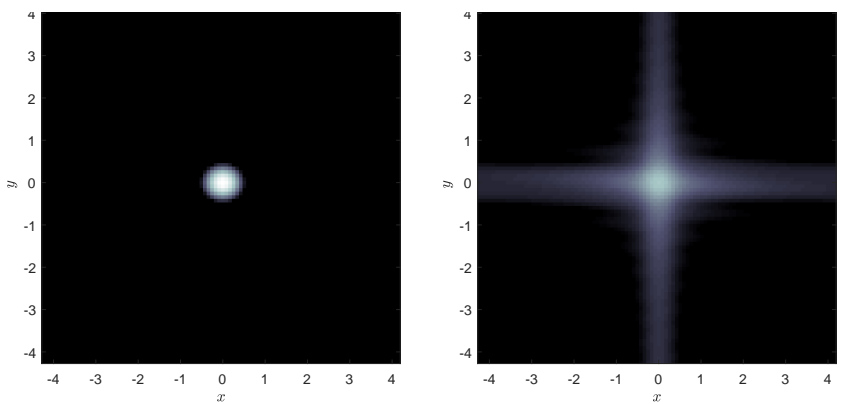

Fig. 5. Example of bright point decomposition. Left: Image $I$. Right: $\mathbf{C}_{1,[1,1]}^{\mathbf{S}, \measuredangle}$ with $R=L=2$.

To limit the side lobes on the wavelet coefficients which are due to the sharp-edge of the Shannon wavelets, we look for alternatives that are subject to smooth transitions. We derive hereafter, a new family of parametrized $R$-band / $L$ look wavelet functions including the Shannon wavelets as limit case.

We propose the following criteria for the design of the new family of wavelets:

- Well located in frequencies and angles (wavelet function).

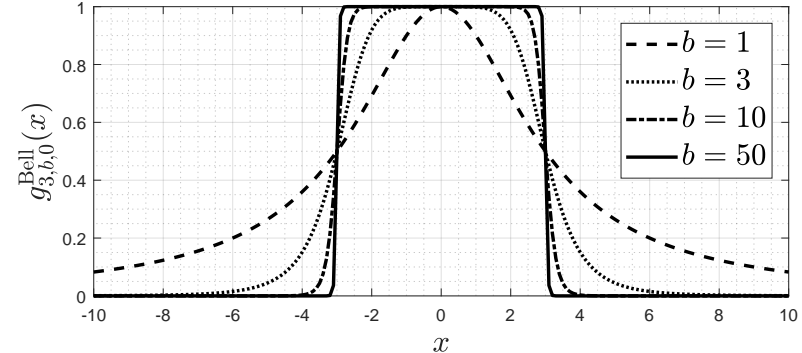

Fig. 6. Bell-shaped function with width $a=3$, center $c=0$ and different slope parameters $b \in\{1,3,10,50\}$.

- Similar behaviour to Shannon wavelets to preserve the framework presented in III-B.

- Smooth transition with a parameter controlling the decay (for adaptability purposes).

Many functions respect the two first criteria. However, Bellshaped membership functions appear to be a good choice as they allow to control both center, extent and slope (and thus smoothness). They are a family of one-dimensional functions defined by:

$$
g_{a, b, c}^{\mathrm{Bell}}(x)=\frac{1}{1+\left|\frac{x-c}{a}\right|^{2 b}} .
$$

where the parameter $a$ stands for the width of the function, where the parameter $b$ controls the slope and the parameter $c$ is a location parameter.

Figure 6 gives an example of Bell function with different slopes and shows that these functions are good candidates for our problem. Using them as a basis, we define:

$$
\Psi_{j,[m, n]}^{\left[d_{1}, d_{2}\right], \measuredangle}(\mathfrak{K}, \theta)=R^{\frac{j}{2}} L^{\frac{j}{2}} H_{j,[m, n]}^{\left[d_{1}, d_{2}\right], \measuredangle}(\mathfrak{K}, \theta) \mathbb{1}_{\mathcal{D}},
$$

where $H_{j,[m, n]}^{\left[d_{1}, d_{2}\right], \measuredangle}$ is defined as a product of two Bell functions:

$$
H_{j,[m, n]}^{\left[d_{1}, d_{2}\right], \measuredangle}(\mathfrak{K}, \theta)=H_{j, m}^{d_{1}, \measuredangle}(\mathfrak{K}) H_{j, n}^{d_{2}, \measuredangle}(\theta)
$$

with:

$$
\begin{aligned}
& H_{j, m}^{d_{1}, \measuredangle}(\mathfrak{K})=g_{\frac{\mathfrak{K}_{B}}{2 R^{j}}, d_{1}, \mathfrak{K}_{0}-\frac{\mathfrak{K}_{B}}{2}+\frac{(2 m+1) \mathfrak{K}_{B}}{2 R^{j}}}^{\text {Bell }}(\mathfrak{K}), \\
& H_{j, n}^{d_{2}, \measuredangle}(\theta)=g_{\frac{\theta_{B}}{L^{j}}, d_{2},-\theta_{B}+\frac{(2 n+1) \theta_{B}}{2 L^{j}}}(\theta) .
\end{aligned}
$$

The definition is similar to that of eq. (10): the center and width of Bell functions have been adapted to span the SAR geometry domain $\mathcal{D}$ through $R^{j}$ translations along $\mathfrak{K}$ and $L^{j}$ translations along $\theta$. The slope parameters $d_{1}$ and $d_{2}$ are let open as a parametrization of the wavelet family.

Define $\Phi_{j,[m, n]}^{\left[d_{1}, d_{2}\right], \measuredangle}=\mathcal{F}^{-1} \Psi_{j,[m, n]}^{\left[d_{1}, d_{2}\right], \measuredangle}(\mathfrak{K}, \theta)$, the wavelet function. We have the following properties:

Proposition III.6 (Vanishing moments). For any non-negative integers $j, m, n, p, q$, we have

$$
\iint_{\mathbb{R}^{2}} x^{p} y^{q} \Phi_{j,[m, n]}^{\left[d_{1}, d_{2}\right], \measuredangle}(x, y) d x d y=0 .
$$

Proof. Similar to III.2. The null derivative in $(0,0)$ is assured by the indicator $\mathbb{1}_{\mathcal{D}}$. 
Proposition III.6 indicates that the functions presented by eq. (16) define wavelets.

Proposition III.7 (Convergence to Shannon wavelets). The $R$ band L-look wavelet transform obtained by using Eq. (16) is associated with the Shannon wavelet transform when $d_{1}, d_{2} \rightarrow$ $+\infty$ :

$$
\lim _{d_{1} \rightarrow+\infty} \lim _{d_{2} \rightarrow+\infty} \Psi_{j,[m, n]}^{\left[d_{1}, d_{2}\right], \measuredangle} \stackrel{\circledast}{=} \Psi_{j,[m, n]}^{\mathrm{S}, \measuredangle} .
$$

where equality holds true almost everywhere (a).

Proof. See Appendix C.

Proposition III.7 highlights that the Bell-shaped wavelets have similar behaviour than Shannon wavelets for high value of $d_{1}$ and $d_{2}$ and can thus be used for analyzing SAR images. For convenience purposes, we use alternatively the notation $\Psi_{j,[m, n]}^{[\infty, \infty]}=\Psi_{j,[m, n]}^{\mathbf{S}, \measuredangle}$.

A problem rises in the choice of these slope parameters. One can intuit that given their value, the properties of orthogonality and completion of wavelet packet subspaces are not assured. Unfortunately, given the expression of the wavelets, finding an interval of values using orthogonality or completion properties is not possible to our knowledge. As such, we propose to consider the wavelet packet in terms of frames (see [49] for details) which relax the conditions of orthogonality and completion. As suggested in [50], a wavelet packet have good reconstruction property if the energy of the signal is preserved when doing the decomposition and reconstruction. In practice, this can be ensured if the following condition is respected [51]:

$$
Q(\mathfrak{K}, \theta)=\sum_{m, n}\left|H_{j,[m, n]}^{\left[d_{1}, d_{2}\right], \measuredangle}(\mathfrak{K}, \theta)\right|^{2} \approx 1, \forall \mathfrak{K}, \forall \theta .
$$

This criterion can be used to grasp qualitatively how the decomposition will treat the frequencies present in the image. If $Q>1$, the energy increase which means that the packet is redundant. When $Q<1$, there is a loss of energy and thus information. We propose to use this criterion to select the values of $d_{1}, d_{2}$ which preserve energy the most.

Since the expression in eq. (17) is separable in $\mathfrak{K}$ and $\theta$, we can treat both separately and solve the problems: $\forall(\mathfrak{K}, \theta) \in \mathcal{D}$, find $d_{1}$ subject to $Q_{\mathfrak{K}}(\mathfrak{K})=\sum_{m, n}\left|H_{j,[m, n]}^{d_{1}, L}(\mathfrak{K})\right|^{2} \approx 1$ and find $d_{2}$ subject to $Q_{\theta}(\theta)=\sum_{m, n}\left|H_{j,[m, n]}^{d_{2}, \measuredangle}(\theta)\right|^{2} \approx 1$.

Figure 7 gives the values of $Q$ for several values of $d_{1}$ and $d_{2}$ for a given set of $\left(j, R, L, f_{0}, B, \theta_{B}\right)$. We notice that for small values of $d_{\epsilon \in\{1,2\}}$, there is a loss of energy at the transitions between the filters. For values of $d_{\epsilon \in\{1,2\}} \in[10, \infty[$, this loss is acceptable. Indeed, since the wavelet packet is developed for target detection schemes, there is a need to know the spectro-angular behaviour in a vector of a fixed size. This means that if the energy of most of each bands are preserved in the coefficients, this will not impact much the detection scheme.

Finally we can compute the wavelets coefficients simply by taking expression at eq. (13) and using $V_{j,[m, n]}(\mathfrak{K}, \theta)=$ $\widetilde{I}(\mathfrak{K}, \theta) H_{j,[m, n]}^{\left[d_{1}, d_{2}\right], \measuredangle}$.
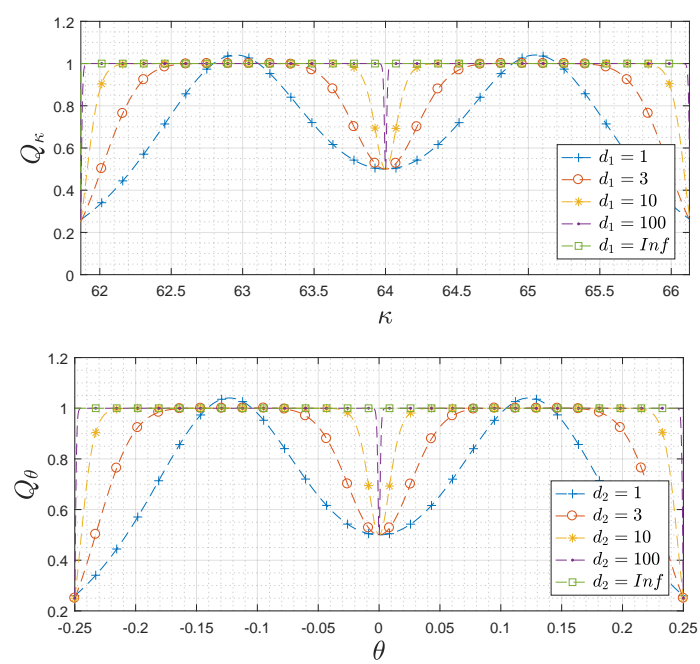

Fig. 7. Redundancy of wavelet packet for $f_{0}=9.6 \mathrm{GHz}, B=640 \mathrm{MHz}, \theta_{B}=0.25$ rad, $j=1, R=L=2$.

\section{Application to target detection}

In this section, we propose to use the wavelet decomposition of the previous section in order to detect a target in a noisy SAR image. First, we give a statistical model for noise disturbances. Then we present the target detection problem.

\section{A. Data Model}

In the following, each pixel location $(p, q)$ of the SAR image will be represented, at a resolution level $j$, by a set of $R$-radius and $L$-look wavelets features encapsulated in the random complex vector

$$
\mathbf{c}_{j}[p, q] \triangleq\left\{\mathbf{C}_{j,[m, n]}^{\left[d_{1}, d_{2}\right], \measuredangle}[p, q]\right\} \begin{aligned}
& m=0,1, \ldots, R^{j}-1 \\
& n=0,1, \ldots, L^{j}-1
\end{aligned} \in \mathbb{C}^{N},
$$

where $N=R^{j} \times L^{j}$.

In standard applications, the vector $\mathbf{c}_{j}[p, q]$ is modeled as a multivariate Gaussian vector: $\mathbf{c}_{j}[p, q]$ follows a Gaussian distribution $\mathcal{C N}(\mathbf{0}, \mathbf{R})$ where $\mathbf{R}$ is the unknown covariance matrix of the data. This model is accurate for SAR images where each pixel is the sum of the contributions of all the scatterers inside its range.

However, when considering HR SAR images, the number of scatterers present in any pixel of the image is small, meaning that the Central Limit Theorem may no longer be applicable. Moreover, there are many non-stationarities inherent to this kind of images where the backscattered power can vary greatly spatially inside the analysis windows. Thus, the Gaussian hypothesis may no longer be applicable. To generalize the Gaussian statistic, we assume that $\mathbf{c}_{j}[p, q]$ follows a CES distribution $\mathcal{C} \mathcal{E}(\mathbf{0}, g, \mathbf{R})$ where the scatter matrix $\mathbf{R}$ is unknown and where $g$ stands for any characteristic function generator [52]. This model extends the Gaussian distribution and better characterizes HR SAR images.

In both models, the matrix $\mathbf{R}$ characterizes the angular and the spectral behaviour of each scatterer. To estimate this matrix, the following $K$ secondary vectors surrounding the 
pixel $(p, q)$ under test (supposed homogeneous in terms of angular and spectral behaviour) are used:

$$
\begin{aligned}
\left\{\mathbf{c}_{j}\left[p-\ell_{1}, q-\ell_{2}\right]\right\} & \\
& \ell_{1}=-K_{1}, \ldots, K_{1} \\
& \ell_{2}=-K_{2}, \ldots, K_{2} \\
& \left(\ell_{1}, \ell_{2}\right) \neq(0,0)
\end{aligned}
$$

with $K=\left(2 K_{1}+1\right)\left(2 K_{2}+1\right)-1$.

We consider two covariance matrix estimators on wavelet feature vectors: the standard Sample Covariance Matrix (SCM) which can be written, under zero-mean wavelet coefficient assumption, in the form

$$
\begin{aligned}
& \hat{\mathbf{R}}_{\mathrm{SCM}, j}[p, q]=\frac{1}{K} \times \\
& \sum_{\substack{\ell_{1}=-K_{1}, \ldots, K_{1} \\
\ell_{2}=-K_{2}, \ldots, K_{2} \\
\left(\ell_{1}, \ell_{2}\right) \neq(0,0)}} \mathbf{c}_{j}\left[p-\ell_{1}, q-\ell_{2}\right] \mathbf{c}_{j}^{H}\left[p-\ell_{1}, q-\ell_{2}\right],
\end{aligned}
$$

and, as alternative to SCM (which can have poorer performance under generalized CES model assumption), the Tyler's Estimator (TE) which has proven some robustness in both Gaussian and non-Gaussian cases and which is defined as the solution of the fixed point equation [53]:

$$
\begin{aligned}
& \hat{\mathbf{R}}_{\mathrm{TE}, j}[p, q]=\frac{N}{K} \times \\
& \quad \sum_{\substack{\ell_{1}=-K_{1}, \ldots, K_{1} \\
\ell_{2}=-K_{2}, \ldots, K_{2} \\
\left(\ell_{1}, \ell_{2}\right) \neq(0,0)}} \frac{\mathbf{c}_{j}^{H}\left[p-\ell_{1}, q-\ell_{2}\right] \hat{\mathbf{R}}_{\mathrm{TE}, j}^{-1}[p, q] \mathbf{c}_{j}\left[p-\ell_{1}, q-\ell_{2}\right]}{} .
\end{aligned}
$$

The TE estimator is robust to non-stationarities that are naturally present in HR SAR images.

For both SCM and TE estimators, the number $K$ has to be around $K \approx 2 N$ for a good estimation [41]. For high values of $R$ or $L$, the vectors become very large. Sometimes, it would be impossible to have sufficient number of secondary samples for the estimation of covariance matrix. In those cases, regularized versions of SCM and TE exist in the literature and have shown good results for many applications [54], [55], [56].

Note that both estimators are used to estimate the covariance matrix of the clutter around a target. Hence, the test pixel (namely $\left(\ell_{1}, \ell_{2}\right) \neq(0,0)$ ) is excluded in the process.

\section{B. Detection Schemes}

We assume that a target with a known steering vector $\mathbf{p} \in \mathbb{C}^{N}$ could be present in some pixels in the SAR image ${ }^{1}$. We have for each pixel $I[p, q]$ to solve the standard binary hypothesis test:

$$
\left\{\begin{array}{lll}
H_{0}: & \mathbf{c}_{j}[p, q]=\mathbf{n}, & \mathbf{c}_{k}=\mathbf{n}_{k} \quad \forall k \in[1, K] \\
H_{1}: & \mathbf{c}_{j}[p, q]=a \mathbf{p}+\mathbf{n}, & \mathbf{c}_{k}=\mathbf{n}_{k} \quad \forall k \in[1, K],
\end{array}\right.
$$

where $\left(\mathbf{n}, \mathbf{n}_{k}\right)$ both represent a noise with the same distribution, $a$ is an unknown complex amplitude of the potential target with spectro-angular steering vector $\mathbf{p}$ to be detected and $\left\{\mathbf{c}_{k}\right\}_{k \in[1, K]}$ being the $K$ secondary data.

\footnotetext{
${ }^{1}$ Note that when the steering vector is not known, it is possible to develop Bayesian target detection schemes using works such as [57], [58].
}

In this detection issue, we decide to test different adaptive detectors like the well-known Adaptive Matched Filter which corresponds to a two-step Generalized Likelihood Ratio Test (GLRT) in homogeneous Gaussian noise [41]):

$$
\Lambda_{j}^{\mathrm{AMF}}[p, q]=\frac{\left|\mathbf{p}^{H} \hat{\mathbf{R}}_{\mathrm{SCM}, j}^{-1}[p, q] \mathbf{c}_{j}[p, q]\right|^{2}}{\mathbf{p}^{H} \hat{\mathbf{R}}_{\mathrm{SCM}, j}^{-1}[p, q] \mathbf{p}} \underset{H_{0}}{\stackrel{H_{1}}{\gtrless}} \lambda,
$$

where $\lambda$ is the detection threshold.

For partially homogeneous Gaussian noise or for CES distributed noises [52], the derivation of the detection problem leads to the Adaptive Normalized Matched Filter [59], [60]:

$$
\begin{aligned}
& \begin{array}{c}
\mathrm{ANMF} \\
\Lambda_{j}[p, q]=
\end{array} \\
& \frac{\left|\mathbf{p}^{H} \hat{\mathbf{R}}_{\mathrm{TE}, j}^{-1}[p, q] \mathbf{c}_{j}[p, q]\right|^{2}}{\left(\mathbf{p}^{H} \hat{\mathbf{R}}_{\mathrm{TE}, j}^{-1}[p, q] \mathbf{p}\right)\left(\mathbf{c}_{j}^{H}[p, q] \hat{\mathbf{R}}_{\mathrm{TE}, j}^{-1}[p, q] \mathbf{c}_{j}[p, q]\right)} \underset{H_{0}}{\stackrel{H_{1}}{\gtrless}} \lambda .
\end{aligned}
$$

The AMF detector has the Constant False Alarm (CFAR) property relative to the Gaussian distribution, while the ANMF is CFAR for both Gaussian and CES distributions. This is an important property since it allows to select a detection threshold to ensure a probability of false alarm $\left(\mathrm{P}_{\mathrm{Fa}}\right)$ independently of the data being tested.

Concerning the complexity of these methods, the limiting factor is the need to compute the inverse of the covariance matrix for both AMF and ANMF schemes. Then, if the number of coefficient is high, this operation becomes time-consuming (typically $\left.O\left((R \times L)^{3}\right)\right)$.

As for the implementation, the target detection schemes can be implemented using parallel computation: by splitting the image in several sub-images and treating each one by a given thread, the computation time is greatly reduced. The simulations presented in section $\mathrm{V}$ were done using a machine with two Intel(R) Xeon(R) CPU E5-2670 v3 @ $2.30 \mathrm{GHz}$ processors, that makes 24 cores in total, and $32 \mathrm{~Gb}$ of RAM, the computation time for an image of size $2510 \times 1638$ and $R=5, L=5$, was 3.35 seconds for the AMF algorithm and 28.34 seconds for the ANMF one.

\section{Simulation and Results}

Some simulation results and discussions on several aspects of wavelet analysis and target detection are presented here.

\section{A. Dataset}

Two dataset have been used to test the wavelet decompositions and their impact on target detection schemes:

- SANDIA Dataset, available at http://www.sandia. gov/radar/complex_data/. The image referenced as MiniSAR20050519p0010image002 is selected.

- SDMS Dataset [61], available at https://www.sdms.afrl.af.mil/ index.php?collection=ccd_challenge. The image referenced as FP0120 is selected.

Table I summarizes the information on both datasets. 


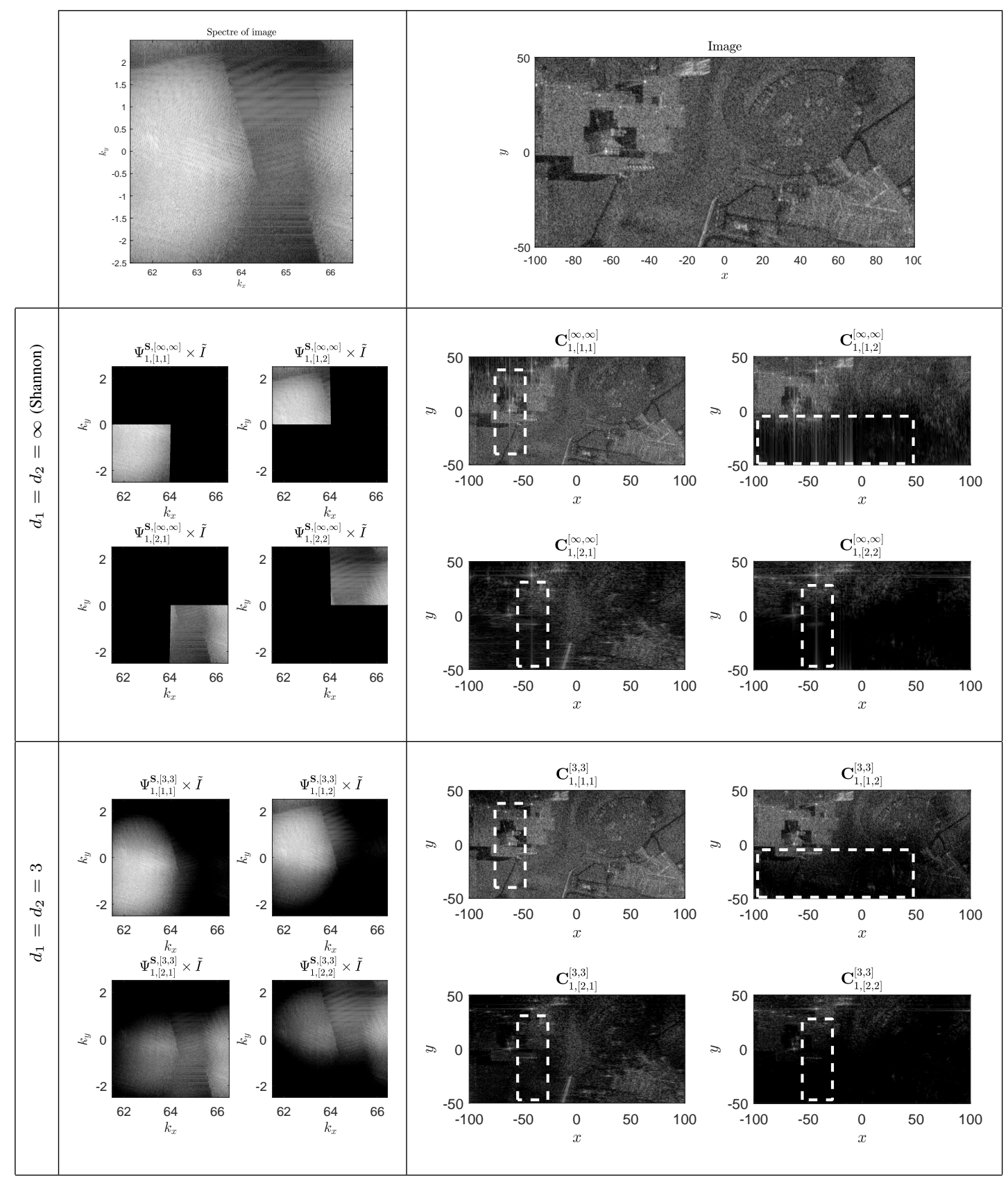

Fig. 8. Coefficients for Shannon and Bell-Shaped wavelets on SDMS Image with $R=2, L=2$. The improvement in terms of linear patterns (side lobes of bright points) for $d_{1}=d_{2}=3$ are highlighted using dashed boxes.

TABLE I

Description of Dataset

\begin{tabular}{lcccc}
\hline Dataset & Band & Frequency & Resolution & Scene description \\
\hline SANDIA & $\mathrm{Ku}$ & $16.8 \mathrm{GHz}$ & $0.10 \mathrm{~m}$ & Stationary aircraft, trees \\
SDMS & $\mathrm{X}$ & $9.6 \mathrm{GHz}$ & $0.20 \mathrm{~m}$ & Foliage, buildings, vehicles \\
\hline
\end{tabular}

\section{B. Simulation description}

For a given SAR image, an artificial target with a given steering vector (representing its spectro-angular behaviour) is embedded. This allows to control both position and Signal to Noise Ratio (SNR) of the target to be detected. For a given image $I$, steering vector $\mathbf{p} \in \mathbb{C}^{R \times L}$, a pixel $\left[i_{t}, j_{t}\right]^{T}$ corresponding to position $\mathbf{r}_{t}=\left[\mathbf{x}\left(i_{t}\right), \mathbf{y}\left(j_{t}\right)\right]^{T}$ and a given $\mathrm{SNR}$ in $\mathrm{dB}$, an image with the target is obtained through:

$$
I_{t}=I+\frac{T}{\|T\|_{l_{2}}} \sigma 10 \frac{\mathrm{SNR}_{\mathrm{dB}}}{20}
$$

with:

$T=\sum_{\substack{m=0, \ldots, R^{j}-1 \\ n=0, \ldots, L^{j}-1}} \mathcal{F}^{-1}\left\{p[m, n] \mathbb{1}_{\Delta_{j, \mathfrak{K}_{m}} \times \Delta_{j, \theta_{n}}} e^{i 2 \pi \mathbf{k}^{T} \mathbf{r}_{t}}\right\}$ and
$\sigma^{2}=\sum_{i=-10, \ldots, 10} I\left(i_{t}+i, j_{t}+i\right)^{2}$, the variance of the noise on a window around the target.

This process is done as follows: 
- Choose a steering vector $\mathbf{p}$.

- Build spectrum according to the steering vector and create image of the targets using eq. (25)

- Perform the wavelet decomposition and create the hyperimage using eq. (13).

- Apply the detectors (23), (24) with the given steering vector.

\section{Results}

1) Spectro-angular behaviour: Figure 8 shows a 2-Band 2Look decomposition of a portion of SDMS Image. First, the spectro-angular behaviour of the data can be analysed: given the sub-image considered, different patterns emerge. Indeed, for example the object in the bottom-right corner $(0>x>$ 100 and $-50<y<0$ ), is not present in the coefficients $\mathbf{C}_{1,[1,2]}$ and $\mathbf{C}_{1,[2,2]}$.

2) Quality of decomposition: Next, the wavelet decomposition is compared with two parameters $d_{1}$ and $d_{2}$. When comparing both Shannon and Bell decomposition in figure 8, we observe for Shannon wavelets linear patterns (side lobes for the strong scatterers present in the scene). When considering $d_{1}=d_{2}=3$, the undesired linear patterns are less prominent. This result was expected as Bell-shaped wavelets make a more concise decomposition in spatial domain.
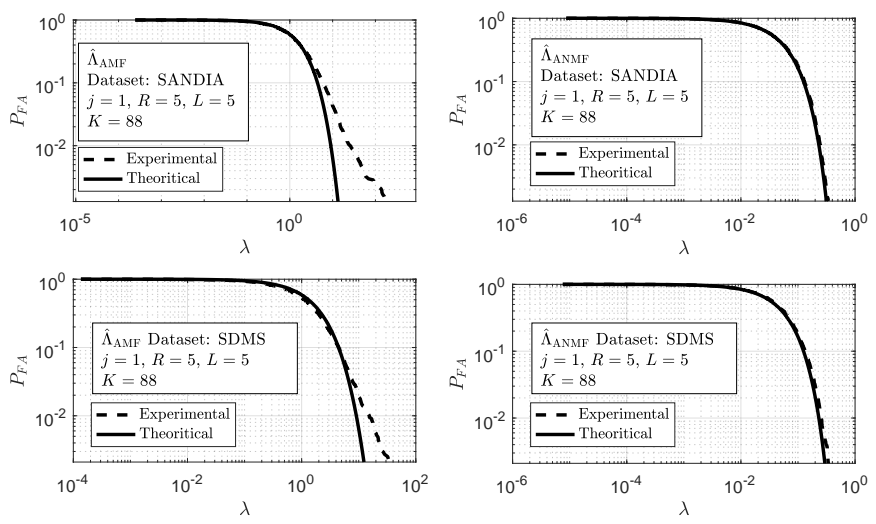

Fig. 9. $P_{F A}-\lambda$ curves. $d_{1}=d_{2}=\infty$. Left: AMF, Right: ANMF. Top: SANDIA dataset, Bottom: SDMS dataset.

3) $P_{F A^{-}} \lambda$ curves: Next, we plot in figure 9 , the $P_{F A^{-}}$ $\lambda$ (probability of false alarm versus threshold of detection) plots for both AMF and ANMF detectors to study the CFAR behaviour of the detectors on the datasets. We choose a random steering vector and apply detectors on the image without any target. It can be observed that the ANMF detector fares a lot better in terms of regulation of false alarm than the AMF. When compared to the theoretical relationship, the AMF detector has an experimental threshold higher at low $P_{F A}$ whereas the ANMF detector stays close to its theoretical performance. This can be interpreted by the heterogeneous nature of the datasets which is not well modeled by Gaussian assumption.

4) Detection near bright point: We choose $R=L=5$ and $j=1$ and we place the target to be detected, with a SNR of $20 \mathrm{~dB}$, near a synthetic bright point with Gaussian spectro-angular behaviour. Figure 10 gives the steering vector of the target to detect, the spectre of both targets and the image obtained by the procedure presented at V-B. The dataset used here is the SANDIA one.
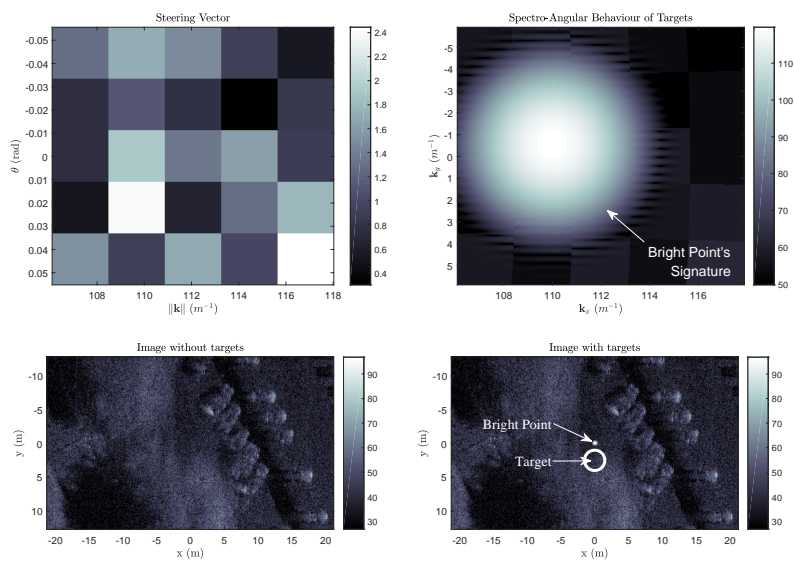

Fig. 10. A target near a bright point. Dataset is SANDIA, $R=L=5$. The target has an amplitude of $-60 \mathrm{~dB}$ when compared to the bright point

Then we apply both detectors on the wavelet coefficients characterized by $d_{1}=d_{2}=\infty$ and $d_{1}=d_{2}=10$. Figure 11 shows the result of the detection at $P_{F A}=10^{-3}$. The threshold guaranteeing the $P_{F A}$ was taken from the experimental curves of figure 9 .
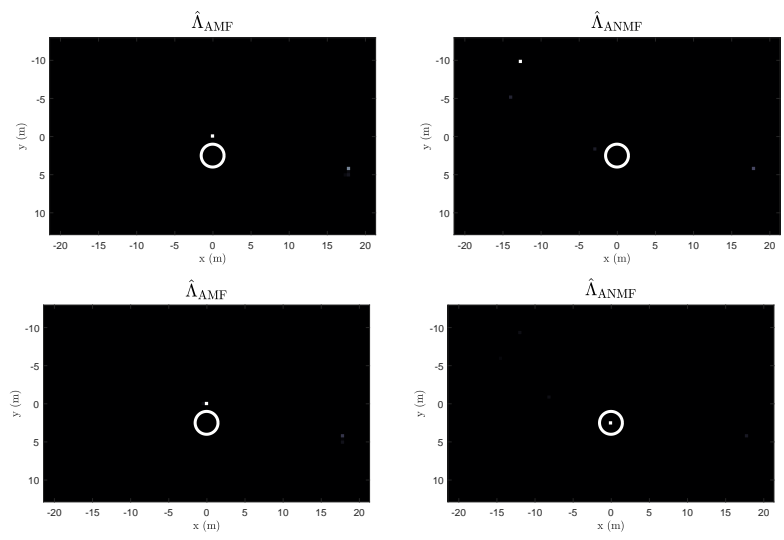

Fig. 11. Results at $P_{F A}=10^{-3}$. Top: $d_{1}=d_{2}=\infty$. Bottom: $d_{1}=d_{2}=$ 10. Dataset is SANDIA, $R=L=5$. The target has an amplitude of $-60 \mathrm{~dB}$ when compared to the bright point.

Discussion: We focus first on the test of detection with $d_{1}=d_{2}=\infty$ (top of the figure). It can be observed that that for both detectors, the target is not detected. The AMF detector gives a false alarm at the position of the bright point, which is expected given that this detector is mostly a powerbased detector. Whereas, the ANMF detector does not detect the bright point as it does not have a similar spectro-angular behaviour as the steering vector. However, a false alarm is still present which can be explained by a similar of the scene signature than the steering vector. If we take a look at detection tests for $d_{1}=d_{2}=10$ (bottom of the figure), we observe that the target is detected with the ANMF detector but not the AMF one. This can be explained by the fact that with $d_{1}=d_{2}=10$, 
we have reduced the side lobes of the bright point which does not pollute the pixel of the target any more resulting in better detection. The AMF detector does not yield better results for the same reason as previously.

These results are interesting since they confirm that the parametrization of the wavelet decomposition impacts the performance of detection.

5) $P_{D}$-SNR Curves: By randomizing the spatial location of the target for Monte Carlo trials, we obtain $P_{D}$-SNR plots for both detectors presented in figure 12. The steering vector is set to a fixed values for all the trials.

Discussion: We first observe that, the ANMF detector performs better in terms of detection than the AMF one for both datasets: if we look at $P_{D}=0.7$, a gain of almost $7 \mathrm{~dB}$ is observed for the ANMF for SDMS dataset and $10 \mathrm{~dB}$ for SANDIA Dataset. This can be interpreted by the non-Gaussian nature of the data which makes regulation of false alarm difficult for the AMF detector and by the fact that ANMF is better suited for heterogeneous data.

The plots for the SANDIA dataset show overall lower performance than the SDMS dataset. This can be explained by the different nature of the datasets: the SANDIA image is more heterogeneous than the SDMS one and the speckle noise is more important.

Next, the different plots for each $d_{1}=d_{2}$, lead to a significant gain when considering $d_{1}=d_{2}=3$ or 10 compared to $d_{1}=d_{2}=\infty$ for the SDMS dataset (about $8 \mathrm{~dB}$ at $P_{D}=0.6$ ). This result is coherent with the observations done previously in $\mathrm{V}-\mathrm{C} 4$. Indeed, the side lobes are contained in the secondary data that is used for the estimation of the covariance matrix. These outliers lead to a loss of accuracy in the estimation which in turn decrease the performances of detection. It can be also observed a loss in detection for $d_{1}=d_{2}=1$. This is coherent with the analysis of the previous section.

The gain using the new wavelet is lower on the SANDIA image (about $1 \mathrm{~dB}$ at $P_{D}=0.6$ ). It is to be expected since the SANDIA dataset contains few bright points spread over the scene.

6) Impact of the steering vector: In order to assess the impact of the steering vector, another Monte-Carlo simulation has been done by setting the SNR to $0 \mathrm{~dB}$ and randomizing the target signature at each trial. For each trial, the target has been set to 100 different location to compute a probability of detection. Table II gives the performance of detection for two values of $d_{1}=d_{2}$ and for both datasets. The same conclusions as previously can be drawn: the ANMF detector performs better than the AMF one on both datasets and using a bellshaped wavelet with a parameter $d_{1}=d_{2}=10$ allows to improve the detection rate.

\section{CONCLUSION}

This paper presented an adaptation of Shannon wavelet packets to SAR geometry in order to retrieve a physical diversity of interest. To reduce the side lobes, which are inherent to wavelet decomposition, a new family of parametrized wavelets has been proposed. These wavelets have the Shannon wavelets as a limit case and are tuned using a redundancy criterion.
This wavelet decomposition has been used in target detection schemes. It has been shown that the spectro-angular diversity, inherent to HR SAR Images, can be used in classic adaptive detection framework. First, the robust framework has proven to be more effective over the Gaussian one in both false alarms regulation and performance of detection. Then, the reduction of side lobes with the new family of wavelets, yields significantly gain in the performance of detection when the image contains numerous bright points.

In this paper, we restrained ourselves to a fixed resolutionlevel for the statistical analysis. It may be interesting to use the multi-resolution framework to selection a decomposition which yields the best possible diversity of a given image while keeping the size of vector low. To this end, a solution can be for example the use of an entropy-based criterion when doing the decomposition.

\section{APPENDIX A}

\section{PROOF OF PROPOSITION III.1}

Proof:

Defining the wavelet coefficients from the following integral

$\mathbf{C}_{j,\left[n_{1}, n_{2}\right]}^{\mathrm{S}}[p, q]=\iint_{\mathbb{R}^{2}} g(z, t) \tau_{\left[M_{1}^{j} p, M_{2}^{j} q\right]} \Phi_{j,\left[n_{1}, n_{2}\right]}^{\mathrm{S}}(z, t) \mathrm{d} z \mathrm{~d} t$.

we have (through Parseval):

$$
\begin{aligned}
\mathbf{C}_{j,[m, n]}^{\mathbf{S}}[p, q] & =\frac{1}{4 \pi} \times \\
& \left.\iint \mathcal{F} g\left(\omega_{1}, \omega_{2}\right) \overline{\mathcal{F} \tau_{\left[M_{1}^{j} p, M_{2}^{j} q\right]} \Phi_{j,[m, n]}^{\mathbf{S}}}\left(\omega_{1}, \omega_{2}\right) \mathrm{d} \omega_{1} \mathrm{~d} \omega_{2}\right) .
\end{aligned}
$$

As (Fourier transform property on the translation)

$$
\begin{aligned}
& \overline{\mathcal{F} \tau_{\left[M_{1}^{j} p, M_{2}^{j} q\right]} \Phi_{j,[m, n]}^{\mathbf{S}}}\left(\omega_{1}, \omega_{2}\right)=e^{i\left(M_{1}^{j} p \omega_{1}\right)} e^{i\left(M_{2}^{j} q \omega_{2}\right)} \times \\
& \mathcal{F} \Phi_{j,[m, n]}^{\mathbf{S}}\left(\omega_{1}, \omega_{2}\right)
\end{aligned}
$$

we obtain:

$$
\begin{aligned}
& \mathbf{C}_{j,[m, n]}^{\mathbf{S}}[p, q]=\frac{1}{4 \pi} \times \\
& \iint e^{i\left(M_{1}^{j} p \omega_{1}\right)} e^{i\left(M_{2}^{j} q \omega_{2}\right)} \mathcal{F} g \mathcal{F} \Phi_{j,[m, n]}^{\mathbf{S}}\left(\omega_{1}, \omega_{2}\right) \mathrm{d} \omega_{1} \mathrm{~d} \omega_{2} .
\end{aligned}
$$

The integral corresponds to the 2D inverse Fourier transform of $[\mathcal{F} g] \Psi_{j,[m, n]}^{\mathbf{S}}$.

\section{APPENDIX B}

PROOFS OF III-B

Proof of Proposition III.2: Function $\Phi_{j,[m, n]}^{\mathbf{S}, \measuredangle}(x, y)$ being separable with respect to $x$ and $y$, we have:

$$
\begin{aligned}
& \iint_{\mathbb{R}^{2}} x^{p} y^{q} \Phi_{j,[m, n]}^{\mathbf{S}}(x, y) \mathrm{d} x \mathrm{~d} y= \\
& \quad-i^{p+q} \frac{\mathrm{d}}{\mathrm{d} \mathfrak{K}^{p}} \frac{\mathrm{d}}{\mathrm{d} \theta^{q}} \Psi_{j,[m, n]}^{\mathbf{S}, \measuredangle}(\mathfrak{K}, \theta) /_{\mathfrak{K}=0, \theta=0}
\end{aligned}
$$

Proposition III.2 follows by noting that $\Psi_{j,[m, n]}^{\mathbf{S}}$ has null derivatives. 

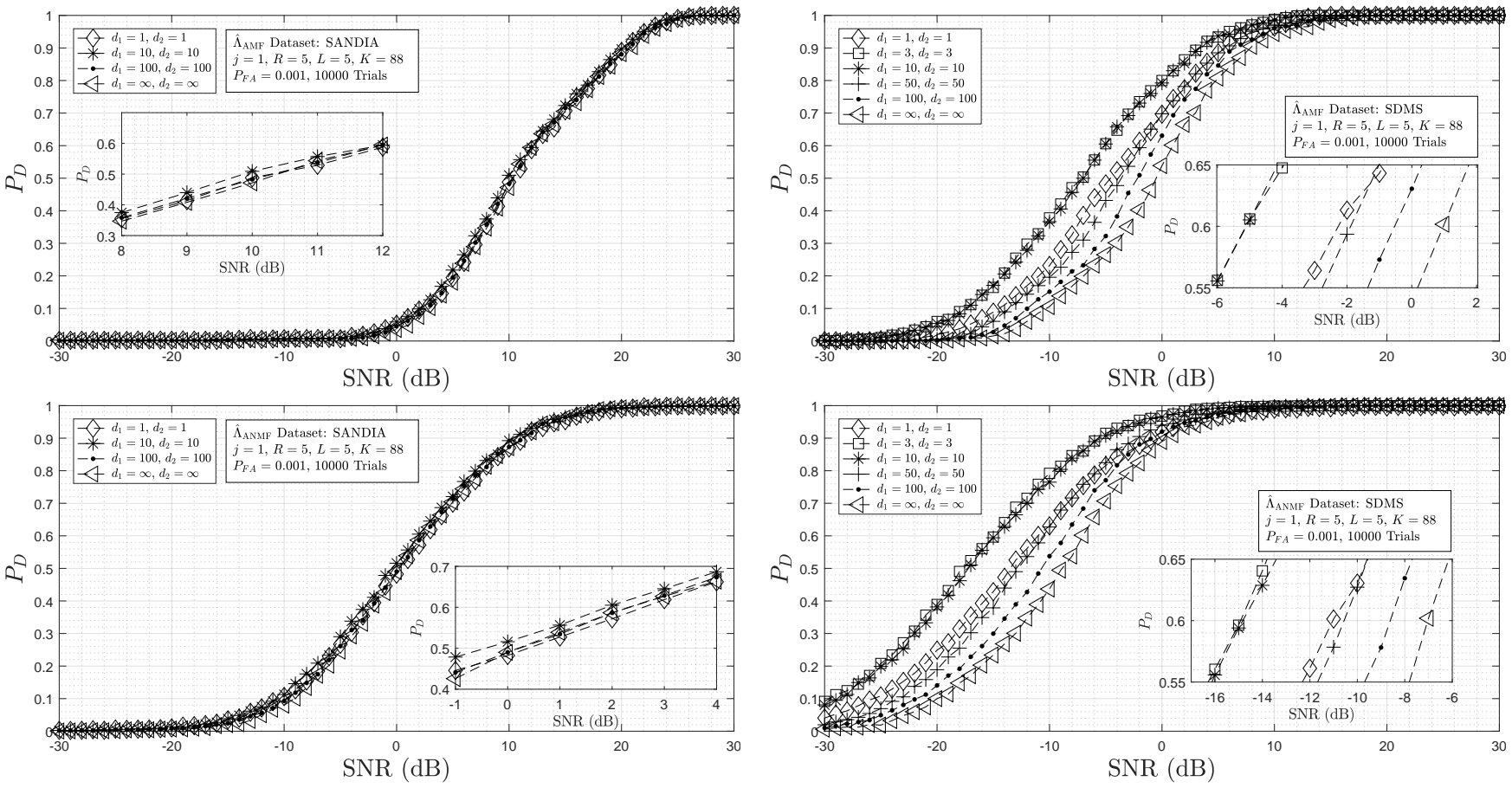

Fig. 12. Pd-SNR plots for several values of $d_{1}=d_{2}$. Top: AMF Detector. Bottom: ANMF Detector.

\begin{tabular}{lllll|llll}
\multicolumn{2}{l}{ SDMS } & & & & SANDIA & & & \\
\hline & & mean & $\min$ & $\max$ & & mean & $\min$ & $\max$ \\
\hline AMF & $d_{1}=10$ & 0.81 & 0.67 & 0.91 & $d_{1}=10$ & 0.04 & 0 & 0.11 \\
& $d_{1}=\infty$ & 0.55 & 0.42 & 0.70 & $d_{1}=\infty$ & 0.02 & 0 & 0.10 \\
\hline \multirow{2}{*}{ ANMF } & $d_{1}=10$ & $\mathbf{0 . 9 5}$ & $\mathbf{0 . 8 9}$ & $\mathbf{1}$ & $d_{1}=10$ & $\mathbf{0 . 5 0}$ & $\mathbf{0 . 3 4}$ & $\mathbf{0 . 7 1}$ \\
& $d_{1}=\infty$ & 0.79 & 0.68 & 0.92 & $d_{1}=\infty$ & 0.46 & 0.22 & 0.66
\end{tabular}

TABLE II

Results of detection when randomising steering vector. SNR=0 dB, 100 different signatures have been tested for 100 different target positions on each image.

Proof of Proposition III.3:

$$
\begin{aligned}
& \left\langle\Phi_{j,[m, n]}^{\mathbf{S}, \measuredangle}, \Phi_{j,\left[m^{\prime}, n^{\prime}\right]}^{\mathbf{S}, \measuredangle}\right\rangle= \\
& \quad \iint_{\mathbb{R}^{2}} \Phi_{j,[m, n]}^{\mathbf{S}, \measuredangle}(x, y) \overline{\Phi_{j,\left[m^{\prime}, n^{\prime}\right]}^{\mathbf{S}, \measuredangle}}(x, y) \mathrm{d} x \mathrm{~d} y
\end{aligned}
$$

By using Parseval formula, we derive

$$
\begin{aligned}
& \left\langle\Phi_{j,[m, n]}^{\mathbf{S}, \measuredangle}, \Phi_{j,\left[m^{\prime}, n^{\prime}\right]}^{\mathbf{S}, \measuredangle}\right\rangle= \\
& \quad \frac{1}{4 \pi^{2}} \iint_{\mathbb{R}^{2}} \mathcal{F} \Phi_{j,[m, n]}^{\mathbf{S}, \measuredangle}(\mathfrak{K}, \theta) \mathcal{F} \Phi_{j,\left[m^{\prime}, n^{\prime}\right]}^{\mathbf{S}, \measuredangle}(\mathfrak{K}, \theta) \mathrm{d} \mathfrak{K} \mathrm{d} \theta .
\end{aligned}
$$

which reduces to

$$
\begin{aligned}
& \left\langle\Phi_{j,[m, n]}^{\mathbf{S}, \measuredangle}, \Phi_{j,\left[m^{\prime}, n^{\prime}\right]}^{\mathbf{S}, \measuredangle}\right\rangle= \\
& \quad \frac{R^{j} L^{j}}{4 \pi^{2}} \iint_{\mathbb{R}^{2}} \mathbb{1}_{\Delta_{j, \mathfrak{K}_{m}} \times \Delta_{j, \theta_{n}}}(\mathfrak{K}, \theta) \mathbb{1}_{\Delta_{j, \mathfrak{K}_{m^{\prime}}} \times \Delta_{j, \theta_{n^{\prime}}}}(\mathfrak{K}, \theta) \mathrm{d} \mathfrak{K} \mathrm{d} \theta .
\end{aligned}
$$

For $m \neq m^{\prime}$ or $n \neq n^{\prime}$, intersection $\Delta_{j, \mathfrak{K}_{m}} \times \Delta_{j, \theta_{n}} \cap \Delta_{j, \mathfrak{K}_{m^{\prime}}} \times$ $\Delta_{j, \theta_{n^{\prime}}}$ of the supports of $\mathcal{F} \Phi_{j,[m, n]}^{\mathbf{S}}$ and $\mathcal{F} \Phi_{j,\left[m^{\prime}, n^{\prime}\right]}^{\mathbf{S}}(\mathfrak{K}, \theta)$ are either disjoint, or reduce to a null set. This ends the proof.
Proof of Proposition III.4: The proof is a consequence of Shannon band-limited function representation and the fact that for any $j \geqslant 1$, the sets $\Delta_{j, \mathfrak{K}_{m}} \times \Delta_{j, \theta_{n}}$, for $m=0,1, \ldots, R^{j}-1$ and $n=0,1, \ldots, L^{j}-1$, are constructed so as to form a partition of $\mathcal{D}$.

\section{APPENDIX C \\ PROOFS OF III-C}

Proof of Proposition III.7:

It suffices to show that $\lim _{d_{1}, d_{2} \rightarrow+\infty} \Psi_{0,[0,0]}^{\left[d_{1}, d_{2}\right], \measuredangle}=\Psi_{0,[0,0]}^{\mathbf{S}, \measuredangle}$. From Eq. (17), we have

$$
H_{0,[0,0]}^{\left[d_{1}, d_{2}\right]}(\mathfrak{K}, \theta)=\frac{1}{1+\left|\frac{2}{\mathfrak{K}_{B}}\left(\mathfrak{K}-\mathfrak{K}_{0}\right)\right|^{2 d_{1}}} \frac{1}{1+\left|\frac{\theta}{\theta_{B}}\right|^{2 d_{2}}}(26)
$$

As a consequence, if $\left|\mathfrak{K}-\mathfrak{K}_{0}\right|<\frac{\mathfrak{K}_{B}}{2}$ and $|\theta|<\theta_{B}$, then:

$$
\lim _{d_{1}, d_{2} \rightarrow+\infty} H_{0,[0,0]}^{\left[d_{1}, d_{2}\right]}(\mathfrak{K}, \theta)=1
$$


In contrast, if $\left|\mathfrak{K}-\mathfrak{K}_{0}\right|>\frac{\mathfrak{K}_{B}}{2}$ or $|\theta|>\theta_{B}$, then:

$$
\lim _{d_{1}, d_{2} \rightarrow+\infty} H_{0,[0,0]}^{\left[d_{1}, d_{2}\right]}(\mathfrak{K}, \theta)=0
$$

which ends the proof.

\section{REFERENCES}

[1] W. G. Carrara, R. S. Goodman, and R. M. Majewski, Spotlight Synthetic Aperture Radar: Signal Processing Algorithms, U. S. Norwood, Ed. Artech House Publishers, 1995.

[2] J. Bertrand and P. Bertrand, "The concept of hyperimage in wide-band radar imaging," Geoscience and Remote Sensing, IEEE Transactions on, vol. 34, no. 5, pp. 1144-1150, Sep. 1996.

[3] M. Duquenoy, J.-P. Ovarlez, L. Ferro-Famil, L. Vignaud, and E. Pottier, "Study of dispersive and anisotropic scatterers behavior in radar imaging using time-frequency analysis and polarimetric coherent decomposition," in Proc. IEEE radar conference, Verona,USA, Apr. 24-27, 2006, pp. $180-185$.

[4] J. Li and E. G. Zelnio, "Target detection with synthetic aperture radar," Aerospace and Electronic Systems, IEEE Transactions on, vol. 32, no. 2, pp. 613-627, April 1996.

[5] Z. Tirandaz and G. Akbarizadeh, "A two-phase algorithm based on kurtosis curvelet energy and unsupervised spectral regression for segmentation of SAR images," Selected Topics in Applied Earth Observations and Remote Sensing, IEEE Journal of, vol. 9, no. 3, pp. 1244-1264, March 2016.

[6] M. Rahmani and G. Akbarizadeh, "Unsupervised feature learning based on sparse coding and spectral clustering for segmentation of synthetic aperture radar images," IET Computer Vision, vol. 9, no. 5, pp. 629-638, 2015.

[7] G. Akbarizadeh, "A new statistical-based kurtosis wavelet energy feature for texture recognition of SAR images," Geoscience and Remote Sensing, IEEE Transactions on, vol. 50, no. 11, pp. 4358-4368, Nov 2012.

[8] D. Karimi, G. Akbarizadeh, K. Rangzan, and M. Kabolizadeh, "Effective supervised multiple-feature learning for fused radar and optical data classification," IET Radar, Sonar and Navigation, vol. 11, no. 5, pp. 768-777, 2017.

[9] G. Akbarizadeh, "A new recognition approach based on genetic algorithm for classifying textures in satellite SAR images," International Journal of Remote Sensing Applications, 2012.

[10] D. Karimi, K. Rangzan, G. Akbarizadeh, and M. Kabolizadeh, "Combined algorithm for improvement of fused radar and optical data classification accuracy," Journal of Electronic Imaging, vol. 26, no. 1, p. 013017, 2017.

[11] H. Rohling, "Radar CFAR thresholding in clutter and multiple target situations," Aerospace and Electronic Systems, IEEE Transactions on, vol. 19, no. 4, pp. 608-621, July 1983.

[12] A. Sharma and R. L. Moses, "Matched subspace detectors for discrimination of targets from trees in SAR imagery," in Conference Record of the Thirty-Fourth Asilomar Conference on Signals, Systems and Computers, vol. 2, Oct 2000, pp. 1721-1726.

[13] A. Izzo, M. Liguori, C. Clemente, C. Galdi, M. D. Bisceglie, and J. J. Soraghan, "Multimodel CFAR detection in foliage penetrating SAR images," Aerospace and Electronic Systems, IEEE Transactions on, vol. 53, no. 4, pp. 1769-1780, Aug 2017.

[14] D. Pastina, P. Lombardo, A. Farina, and P. Daddi, "Super-resolution of polarimetric SAR images of ship targets," Signal Processing, vol. 83, no. 8, pp. $1737-1748,2003$.

[15] A. Alonso-Gonzalez, C. Lopez-Martinez, and P. Salembier, "Filtering and segmentation of polarimetric SAR data based on binary partition trees," Geoscience and Remote Sensing, IEEE Transactions on, vol. 50, no. 2, pp. 593-605, Feb 2012.

[16] D. Ratha, A. Bhattacharya, and A. C. Frery, "Unsupervised classification of PolSAR data using a scattering similarity measure derived from a geodesic distance," IEEE Geoscience and Remote Sensing Letters, vol. 15, no. 1, pp. 151-155, Jan 2018.

[17] F. Bandiera, M. Jahangir, G. Ricci, and R. Verrienti, "Adaptive radar detection without secondary data: Exploiting a diversity idea," in 2006 14th European Signal Processing Conference, Sept 2006, pp. 1-5.

[18] D. Cerutti-Maori, I. Sikaneta, and C. H. Gierull, "Optimum SAR/GMTI processing and its application to the radar satellite RADARSAT-2 for traffic monitoring," Geoscience and Remote Sensing, IEEE Transactions on, vol. 50, no. 10, pp. 3868-3881, Oct 2012.
[19] S. X. Zhang, M. D. Xing, X. G. Xia, R. Guo, Y. Y. Liu, and Z. Bao, "Robust clutter suppression and moving target imaging approach for multichannel in azimuth high-resolution and wide-swath synthetic aperture radar," Geoscience and Remote Sensing, IEEE Transactions on, vol. 53, no. 2, pp. 687-709, Feb 2015.

[20] D. Li, M. Zhan, J. Su, H. Liu, X. Zhang, and G. Liao, "Performances analysis of coherently integrated CPF for LFM signal under low SNR and its application to ground moving target imaging," Geoscience and Remote Sensing, IEEE Transactions on, vol. 55, no. 11, pp. 6402-6419, Nov 2017.

[21] B. Liu, K. Yin, Y. Li, F. Shen, and Z. Bao, "An improvement in multichannel SAR-GMTI detection in heterogeneous environments," Geoscience and Remote Sensing, IEEE Transactions on, vol. 53, no. 2, pp. 810-827, Feb 2015.

[22] I. C. Sikaneta and C. H. Gierull, "Adaptive CFAR for space-based multichannel SAR-GMTI," Geoscience and Remote Sensing, IEEE Transactions on, vol. 50, no. 12, pp. 5004-5013, Dec 2012.

[23] A. Taylor, P. Forster, F. Daout, H. M. Oriot, and L. Savy, "A generalization of the Fixed Point estimate for packet-scaled complex covariance matrix estimation," Signal Processing, IEEE Transactions on, vol. 65, no. 20, pp. 5393-5405, Oct 2017.

[24] E. P. Simoncelli, W. T. Freeman, E. H. Adelson, and D. J. Heeger, "Shiftable multiscale transforms," Information Theory, IEEE Transactions on, vol. 38, no. 2, pp. 587-607, March 1992.

[25] M. Unser, N. Chenouard, and D. Van De Ville, "Steerable pyramids and tight wavelet frames in," Image Processing, IEEE Transactions on, vol. 20, no. 10, pp. 2705-2721, Oct 2011.

[26] E. Candès, L. Demanet, D. Donoho, and L. Ying, "Fast discrete curvelet transforms," Multiscale Modeling \& Simulation, vol. 5, no. 3, pp. 861899, 2006.

[27] R. Durand, G. Ginolhac, L. Thirion, and P. Forster, "New SAR processor based on matched subspace detectors," Aerospace and Electronic Systems, IEEE Transactions on, vol. 45, no. 1, pp. 221-236, Jan 2009.

[28] F. Brigui, L. Thirion-Lefevre, G. Ginolhac, and P. Forster, "New SAR algorithm based on orthogonal projections for MMT detection and interference reduction," Geoscience and Remote Sensing, IEEE Transactions on, vol. 52, no. 7, pp. 3800-3811, July 2014.

[29] C. Brekke, S. N. Anfinsen, and Y. Larsen, "Subband extraction strategies in ship detection with the subaperture cross-correlation magnitude," IEEE Geoscience and Remote Sensing Letters, vol. 10, no. 4, pp. 786790, July 2013.

[30] G. D. De Grandi, J. S. Lee, and D. L. Schuler, "Target detection and texture segmentation in polarimetric SAR images using a wavelet frame: Theoretical aspects," Geoscience and Remote Sensing, IEEE Transactions on, vol. 45, no. 11, pp. 3437-3453, Nov 2007.

[31] G. D. De Grandi, R. M. Lucas, and J. Kropacek, "Analysis by wavelet frames of spatial statistics in SAR data for characterizing structural properties of forests," Geoscience and Remote Sensing, IEEE Transactions on, vol. 47, no. 2, pp. 494-507, Feb 2009.

[32] S. Zecchetto and F. D. Biasio, "A wavelet-based technique for sea wind extraction from SAR images," Geoscience and Remote Sensing, IEEE Transactions on, vol. 46, no. 10, pp. 2983-2989, Oct 2008.

[33] C. Lopez-Martinez and X. Fabregas, "Modeling and reduction of SAR interferometric phase noise in the wavelet domain," Geoscience and Remote Sensing, IEEE Transactions on, vol. 40, no. 12, pp. 2553-2566, Dec 2002.

[34] J. Ma, M. Gong, and Z. Zhou, "Wavelet fusion on ratio images for change detection in SAR images," IEEE Geoscience and Remote Sensing Letters, vol. 9, no. 6, pp. 1122-1126, Nov 2012.

[35] N. S. Subotic, L. M. Collins, J. D. Gorman, and B. J. Thelen, "A multiresolution approach to target detection in synthetic aperture radar data," in Proceedings of 1994 28th Asilomar Conference on Signals, Systems and Computers, vol. 1, Oct 1994, pp. 122-126.

[36] W. W. Irving, L. M. Novak, and A. S. Willsky, "A multiresolution approach to discrimination in SAR imagery," Aerospace and Electronic Systems, IEEE Transactions on, vol. 33, no. 4, pp. 1157-1169, Oct 1997.

[37] M. Tria, J.-P. Ovarlez, L. Vignaud, J. C. Castelli, and M. Benidir, "Discriminating real objects in radar imaging by exploiting the squared modulus of the continuous wavelet transform," IET Radar, Sonar and Navigation, vol. 1, no. 1, pp. 27-37, Feb. 2007.

[38] J.-P. Ovarlez, L. Vignaud, J. C. Castelli, M. Tria, and M. Benidir "Analysis of SAR images by multidimensional wavelet transform," IEE Proceedings - Radar, Sonar and Navigation, vol. 150, no. 4, pp. 234241, Aug. 2003.

[39] J.-P. Ovarlez, G. Ginolhac, and A. M. Atto, "Multivariate linear timefrequency modeling and adaptive robust target detection in highly textured monovariate SAR image," in 2017 IEEE International Conference 
on Acoustics, Speech and Signal Processing (ICASSP), March 2017, pp. 4029-4033.

[40] A. Mian, J.-P. Ovarlez, G. Ginolhac, and A. M. Atto, "Multivariate change detection on high resolution monovariate SAR image using linear Time-Frequency analysis," in 25th European Signal Processing Conference (EUSIPCO), Kos, Greece, Aug. 2017.

[41] F. C. Robey, D. R. Fuhrmann, E. J. Kelly, and R. Nitzberg, "A CFAR adaptive matched filter detector," Aerospace and Electronic Systems, IEEE Transactions on, vol. 28, no. 1, pp. 208-216, Jan 1992.

[42] S. Kraut and L. L. Scharf, "The CFAR adaptive subspace detector is a scale-invariant GLRT," Signal Processing, IEEE Transactions on, vol. 47, no. 9, pp. 2538-2541, Sep 1999.

[43] E. Conte, M. Lops, and G. Ricci, "Adaptive detection schemes in compound-Gaussian clutter," Aerospace and Electronic Systems, IEEE Transactions on, vol. 34, no. 4, pp. 1058-1069, Oct 1998.

[44] L. Denis, A. Ferrari, D. Mary, L. Mugnier, and E. Thiébaut, "Fast and robust detection of a known pattern in an image," in 24th European Signal Processing Conference (EUSIPCO), Aug 2016, pp. 2206-2210.

[45] M. Duquenoy, J.-P. Ovarlez, L. Ferro-Famil, E. Pottier, and L. Vignaud, "Scatterers characterisation in radar imaging using joint time-frequency analysis and polarimetric coherent decompositions," IET Radar, Sonar and Navigation, vol. 4, no. 3, pp. 384-402, June 2010.

[46] N. Hess-Nielsen, "Control of frequency spreading of wavelet packets," Applied and Computational Harmonic Analysis, vol. 1, no. 2, pp. 157168, March 1994.

[47] M. Nielsen, "Highly nonstationary wavelet packets," Applied and Computational Harmonic Analysis, vol. 12, no. 2, pp. 209-229, March 2002.

[48] A. Averbuch, R. R. Coifman, D. Donoho, M. Elad, and M. Israeli, "Fast and accurate polar Fourier transform," Applied and Computational Harmonic Analysis, vol. 21, no. 2, pp. 145 - 167, 2006.

[49] O. Christensen, An Introduction to Frames and Riesz Bases, ser. Applied and Numerical Harmonic Analysis. Birkhäuser Boston, 2002.

[50] A. Cohen, I. Daubechies, and J. Feauveau, "Biorthogonal bases of compactly supported wavelets," Communications on Pure and Applied Mathematics, vol. 45, no. 5, pp. 485-560, Jun 1992.

[51] J. Kovacevic, P. L. Dragotti, and V. K. Goyal, "Filter bank frame expansions with erasures," Information Theory, IEEE Transactions on, vol. 48, no. 6, pp. 1439-1450, Jun 2002.

[52] E. Ollila, D. E. Tyler, V. Koivunen, and H. V. Poor, "Complex Elliptically Symmetric distributions: Survey, new results and applications," Signal Processing, IEEE Transactions on, vol. 60, no. 11, pp. 5597 -5625, nov. 2012.

[53] F. Pascal, Y. Chitour, J.-P. Ovarlez, P. Forster, and P. Larzabal, "Covariance structure maximum likelihood estimates in compound Gaussian noise: Existence and algorithm analysis," Signal Processing, IEEE Transactions on, vol. 56, no. 1, pp. 34-38, January 2008.

[54] Y. Chen, A. Wiesel, and A. O. Hero, "Robust shrinkage estimation of high-dimensional covariance matrices," Signal Processing, IEEE Transactions on, vol. 59, no. 9, pp. 4097-4107, 2011.

[55] F. Pascal, Y. Chitour, and Y. Quek, "Generalized robust shrinkage estimator and its application to STAP detection problem," Signal Processing, IEEE Transactions on, vol. 62, no. 21, pp. 5640-5661, Nov 2014.

[56] E. Ollila and D. E. Tyler, "Regularized M-estimators of scatter matrix," Signal Processing, IEEE Transactions on, vol. 62, no. 22, pp. 60596070, Nov 2014.

[57] O. Besson, A. Coluccia, E. Chaumette, G. Ricci, and F. Vincent, "Generalized likelihood ratio test for detection of Gaussian rank-one signals in Gaussian noise with unknown statistics," Signal Processing, IEEE Transactions on, vol. 65, no. 4, pp. 1082-1092, Feb 2017.

[58] A. Coluccia and G. Ricci, "Adaptive radar detectors for point-like Gaussian targets in Gaussian noise," Aerospace and Electronic Systems, IEEE Transactions on, vol. 53, no. 3, pp. 1284-1294, June 2017.

[59] S. Kraut, L. Scharf, and L. M. Whorter, "Adaptive Subspace Detector," Signal Processing, IEEE Transactions on, vol. 49, no. 1, pp. 1-16, Jan 2001.

[60] M. S. Greco and A. De Maio, Eds., Modern Radar Detection Theory. SciTech Publishing, Jan 2016.

[61] S. M. Scarborough, L. Gorham, M. J. Minardi, U. K. Majumder, M. G. Judge, L. Moore, L. Novak, S. Jaroszewksi, L. Spoldi, and A. Pieramico, "A challenge problem for SAR change detection and data compression," in Proc. of SPIE, Algorithms for Synthetic Aperture Radar Imagery XVII, vol. $7699,2010$.

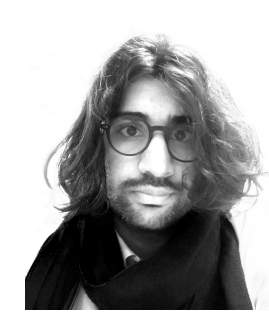

Ammar Mian (S' 18) received the Master's degree in Signal Processing from the Grenoble-INP University, France in 2016. He is currently working toward the Ph.D. degree in Signal Processing. from the CentraleSupélec Univeristy, France. His research interests are mainly in the field of Statistical Signal Processing with a focus on the processing of remotely sensed images. Others topics of interests include Time-Frequency Analysis and Machine Learning.

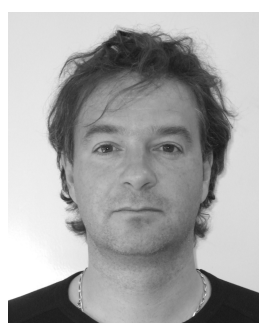

Jean-Philippe Ovarlez (M'06) was born in Denain, France in 1963. He received jointly the engineering degree from Ecole Supérieure d'Electronique Automatique et Informatique (ESIEA), Paris, France and the Diplôme d'Etudes Approfondies degree in Signal Processing from University of Paris XI, Orsay, France and the Ph.D. degree in Physics from the University of Paris VI, Paris, France, in 1987 and 1992, respectively. In 2011, he obtained a Research Directorship Habilitation (HDR) thesis in Signal Processing from the University of Paris-Sud and his qualification to the University Professor position. In 1992, he joined the Electromagnetic and Radar Division of the French Aerospace Lab (ONERA), Palaiseau, France, where he is currently Chief Scientist and member of the Scientific Committee of the ONERA Physics Branch. Since 2008, he is attached at a part time to Centrale-Supélec SONDRA Lab, in charge of Signal Processing activities supervision. In 2015, he becomes member of Special Area Team (SAT) in Theoretical and Methodological Trends in Signal Processing (TMTSP), EURASIP and treasurer of the IEEE GRSS French Chapter in 2016. His research interests are centered in the topic of Statistical Signal Processing for radar and SAR applications such as Time-Frequency, imaging, detection and parameters estimation.

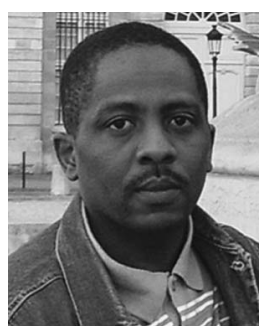

Abdourrahmane Mahamane Atto (M'09,SM'16) received the Master's degrees in applied mathematics and pure mathematics from the University of Abomey-Calavi, Abomey Calavi, Benin, in 2001 and 2002, respectively; the Master's degree in advanced studies in electrical engineering from école Polytechnique d'Abomey-Calavi, Abomey Calavi, in 2002; the Master's degree in image and artificial intelligence from TELECOM Bretagne, Brest, France, in 2005; the Ph.D. degree in applied mathematics from the University of Rennes I, Rennes, France, and TELECOM Bretagne in 2008; and the Habilitation degree for research supervision from Université Grenoble Alpes, Grenoble, France, in 2015.

From 2002 to 2003, he worked with école Polytechnique d'Abomey- Calavi; from 2003 to 2004, with école des Mines, de l'Industrie et de la Géologie, Niamey, Niger; from 2008 to 2009, with TELECOM Bretagne; and from 2009 to 2011, with Institut Polytechnique de Bordeaux, Talence, France. Since September 2011, he has been an Associate Professor with Polytech Annecy-Chambéry, Université Savoie Mont Blanc, Annecy-Le-Vieux, France. His research interests include mathematical methods and models for signal, image, and information processing.

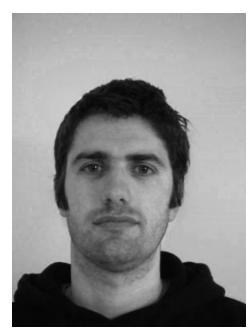

Guillame Ginolhac (S'98,M'01,SM'12) received the Master's degree of Electrical Engineer, in 1997 and the Ph.D. degree of Signal Processing, in 2001 both from the Grenoble-INP university (France). From 2002 to 2012, he was an associate professor in SATIE Lab and University Paris Nanterre. In 2011, he obtained a Research Directorship Habilitation (HDR) thesis in Signal Processing from the ENS Cachan institute. From 2012, Guillaume Ginolhac is a full professor in LISTIC Lab at University Savoie Mont-Blanc. From July 2018, he is the head of the "Connaissances, Images, Télédétection" group of LISTIC. His research interests include estimation and detection theory for statistical signal processing and applications to array processing and radar/sonar. 